\title{
Effective Use of Dental Implants and Prosthetics for Dental Missions: Clinical Case Presentations
}

\author{
Michael A Fisher ${ }^{1}$ and Charles J Arcoria ${ }^{2 *}$ \\ ${ }^{1}$ Private Practice, USA \\ ${ }^{2}$ A.T. Still University, USA
}

Submission: April 18, 2015; Published: May 05, 2016

*Corresponding author: Charles J Arcoria, DDS, MBA, EA, Adjunct Professor, A.T. Still University, 12884 Coronado Drive Freeport, TX 77541 , USA, Tel: 8176800239; Email: charlesarcoria@gmail.com

\begin{abstract}
Dentists practicing in a mission setting have limited options for comprehensive treatment of patients because of the distance from the full resources available in conventional dental office setting. Because of the limited time aspect of team mission work, emergency extractions and limited operative treatment are typically performed. Inherently, the lack of follow-up care with extensive restorations and the need for proper maintenance, preclude many treatment modalities. As a result, this efficient but not simplistic model of mission dentistry is designed to deliver treatment that can be quickly accomplished with recurrent follow-up over a series of eight week time frames. These three clinical case presentations document the type of functional and esthetic treatment that can be accomplished with intermittent and minimal appointments scheduled over an interval of less than one year. This treatment concept depicts complete dentures retained with a minimal number of implants, bypassing the traditional Operative Dentistry procedures which in these types of cases cannot be easily maintained. The contrast of restoring severely decayed tooth structure vs. extracting those teeth which have a low probability of long-term survival, sets the basis for the combined maxillary-mandibular dental implants and prosthetics treatment plan. Designed to be used as a template for many adult patients, the mission dentist has an avenue for helping patients achieve optimum health, with reduced resources and costs. These clinical case presentations will demonstrate the: 1) effective use of multiple extractions, 2) precision alveoloplasty, 3) immediate implant placement in strategic locations in the mandibular and maxillary arches, and 4) placement of maxillary and mandibular dentures over the existing implants. Combining optimum prosthetics and esthetics, the emergency patient in a mission setting can obtain results that are both comprehensive and sustainable.

Keywords: Implants; Prosthetics; Dentsply
\end{abstract}

\section{Introduction}

How does the mission dentist develop and administer effective and long-lasting treatment plans for patients that are seen approximately three of four times in the span of one year [1]. This is the perplexing question that mission dentists have struggled with for decades. The effort to help indigent patients in distant locations who have minimal access to follow-up care, once the dental mission team has departed, is a crucial problem [2,3]. Because of the greatly improved success rates of placing and restoring dental implants within the past ten years, unsophisticated and uncomplicated treatment regimens which potentially bypass traditional dental care can now be frequently employed for even indigent patients. As a result, mission dentistry treatment planning must now encompass a more efficient method to insure longevity of restorative work to be undertaken. But, what can actually be accomplished involving intermittent intervals over the duration of one calendar year? It is acknowledged that the placement of multiple restorations in severely decayed teeth, without the availability for possible Endodontic and/or Periodontal follow-up treatment is a prescription for eventual failure. Practitioners have experienced a form of this problem during their undergraduate curricula, but not by design [4]. Phenomenal efforts were undertaken to "save" teeth at all costs, which is not a dishonorable pursuit. However, what commenced as a sizeable class II restoration on a permanent mandibular first molar, progressed to a full crown, then Endodontic therapy with a second crown, then Periodontal treatment to address the osseous support problems, and finally extraction as the unrestorable tooth has served its usefulness over the span of approximately five years in the dental education of several students [5]. But, is this really the best approach for the indigent patient that deserves better? Mission practitioners may need to discard many types of Operative and Endodontic treatment regimens that could only be successfully conducted within a traditional dental office setting. If the clinician were to be relieved of the burden that all teeth in all conditions in all patients must be saved at all costs, then comprehensive dental treatment could be accomplished with multiple tooth extractions, placement of dental implants in strategic locations, and secured with maxillary and mandibular dentures. Would this not be an acceptable form of long-term treatment for many 
indigent patients in a mission setting? Understandably, this new mission treatment regimen is more complicated instead of placing multiple restorations in severely compromised teeth or undertaking extensive Endodontic and Periodontal therapy. But, the long-term functioning capabilities and prosthesis management by the patient easily can surpass this problem. Many of the same principles and procedures we use in traditional implant dentistry in the private setting, with respect to implantsupported over-dentures and implant-retained hybrid bridges, can also be applied to domestic and international dental mission endeavors. The mini implants so often relied on to retain transitional prostheses while waiting for extraction sites and alveoloplasty to heal, and conventional implants to integrate, can also be considered as "permanent" supports for chair-side relined and retained transitional dentures when further care is limited by inaccessibility to conventional dental labs, or inability for further appointments. Thus, in a missional setting, whether domestic or international, if a minimum of three appointments can be assured within the course of six months to a year, then a transitional denture can be fabricated at the dentist's lab of choice after only a single set of impressions during a first visit. Subsequently, at a second visit (whether by the original dentist or another from the same or different ministry) extractions, alveoloplasty and mini implant placement can be accomplished, with initial reline and stabilization of the transitional denture. Finally, at a third visit after complete tissue healing, a final chair side reline can be performed to re-adapt the mini implant retainers if needed, and contour tissue surfaces to their most ideal form. If no further treatment is possible then the dentist can take comfort knowing that the patient will be in a much healthier state, have a much more functional dentition, and have a significantly improved sense of self-esteem than when first encountered. Should circumstances change in the future, whether with respect to patient financial ability when dealing with domestic mission endeavors, or patient availability and proximity when care has been undertaken internationally, then such cases can be "completed" in the conventional sense with additional conventional implants and over-dentures or hybrid bridges as seen in the following cases.

\section{Patient Selection and Evaluation}

With this initial concept, it is important that the proper selection of patients be undertaken. Careful triage of patients during the initial mission dentistry experience will determine the best use of time and also the most optimum form of treatment. Substituting extractions for Operative preparations that are destined to fail in a short period of time, determine the type of implant placement and prosthetics that will be placed at a later date. Treatment can be accomplished by utilizing the following approach:
4.1. Step 1: Triage teeth regarding the potential to salvage, or to extract.

\subsection{Step 2: Alveoplasty select areas.}

4.3. Step 3: Mandibular implant placement.

4.4. Step 4: Prosthetic construction and placement.

For these patients, when the teeth are extracted, a precise and profound alveoloplasty will be performed, in all dimensions [6]. Clinicians should consider an alveoloplasty procedure as possessing a combination of artistic and mechanical methodologies. Mechanically, it is incumbent upon the dentist to smooth and level the osseous tissue to remove sharp and irregular projections $[7,8]$. In addition, it is just as important to reduce the height of the bone in each arch, superiorly on the maxilla and inferiorly on the mandible so that there is sufficient room subsequently for the prosthetics. This necessary dimension (inter-arch space), and properly planning for it, and actually purposefully creating it, is absolutely vital to the success of any combination dental implant and prosthetic case. This allows for adequate inter-arch space and sufficient room for the necessary thickness of an attractive and properly proportioned set of dentures [9].

\section{Clinical Case Presentations}

\section{Case 1}

Dale is a 52 year old male who stated: "I want to fix my teeth and have a great smile when I'm done!" He was missing a number of teeth, and many that were remaining in his mouth had failing restorations, and/or long-term poor periodontal prognoses (Figure 1A-1E). After reviewing radiographs and digital photographs, implants were not an option to replace the patient's existing missing teeth due to severe atrophy in all edentulous areas, and extensive sinus pneumatization in the left maxilla (Figure 2). Due to loss of the posterior teeth intercuspal position centric stops and excessive attrition of the remaining anterior teeth, the patient exhibited posterior occlusal collapse, with approximately $5 \mathrm{~mm}$ of lost vertical dimension. The patient did not wish to consider "patch and fix" treatment, nor did he wish to replace current or future missing teeth with removable partial dentures. After thoroughly discussing other options, which potentially included complete dentures, implantsupported over-dentures, and implant-retained hybrid fixed prostheses, the patient requested removal of the remaining teeth and replacement with four implants in each arch to anchor screwretained hybrid bridges [10]. Once implant-supported prostheses were chosen, the option of conventional sinus grafts to allow placement of implants in the molar regions was discussed. The patient did not wish to pursue this regimen, due to the potential morbidity of the procedure itself (and the associated cost) or 
the additional healing time for graft maturation (traditionally six months prior to implant placement). Consequently, it was opted to place four implants in each arch, and incline the two maxillary posterior implants at approximately 25 degrees distally to disengage from the maxillary sinuses. Similarly, the mandibular posterior implants were inclined approximately 25 degrees distally to separate from the mental foramina and the small, but present, anterior loops of the mental nerves. The patient elected not to immediately place provisional restorations for the implants (as is commonly performed with the All-onFour, and similar techniques) because it was desired to maintain optimal hygiene throughout the healing phase. The patient believed he could perform that much better if the provisional prostheses were removed, as needed. He also desired to lessen any potential risk factors such as post-operative infection around his implants, or mechanical overload (since he exhibited an aggressive bruxism habit), by completely submerging them beneath soft tissue during their integration period. As a result, conventional maxillary and mandibular transitional dentures were fabricated, and mini implants (MDI by 3M) placed to help stabilize and retain his mandibular transitional prosthesis throughout the treatment process. Treatment was initiated by extracting the patient's maxillary teeth without complication, and performing judicious alveoloplasty to smooth and level his remaining osseous ridge prior to implant placement (Figure 3A\&3B). Care was given to removing sufficient osseous height to allow room for the necessary layers of materials and components required by a hybrid bridge (teeth, acrylic gum tissue, supportive framework and implant abutments), though it's worth noting that some of the inter-arch space required was actually gained by opening the occlusal contacting space dimension approximately $5 \mathrm{~mm}$ ( $2.5 \mathrm{~mm}$ per arch) to regain ideal vertical dimension. Once the osseous ridge was prepared, the posterior implant positions were sited using previous tooth positions as landmarks (Figure 4A\&4B) (Figure 5). Initial osteotomies were prepared following the expected medial wall positions and angulation of the sinuses and paralleling pins were placed with digital radiographs taken to verify the position and angulation. It is worth noting that paralleling pins are significantly narrower than the final implants so care was taken to ensure that there was sufficient room distal to the paralleling pins to allow enlargement of the osteotomies without perforating the medial sinus walls. Once the posterior implants were sited, the anterior implants were located in the lateral incisor positions, and their osteotomies were directed towards the palatal aspects of the sockets to engage the thicker, denser portion of the alveolus. All four implants (ASTRA TECH EV by Dentsply, $4.2 \times 11 \mathrm{~mm}$ ) were placed at 45 newton centimeter $(\mathrm{N} \bullet \mathrm{cm})$, following the manufacturer's protocol of sequentially enlarging the osteotomies, and using copious sterile saline irrigation (Figure 6A-6D).

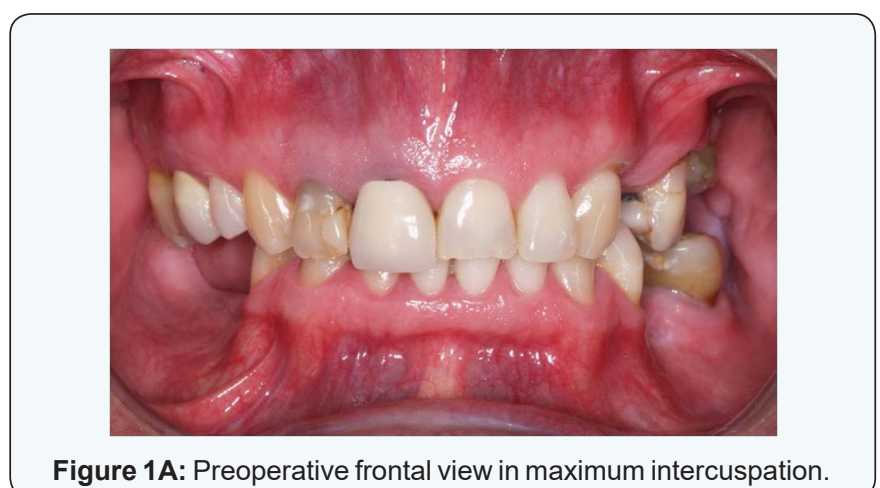

Figure 1A: Preoperative frontal view in maximum intercuspation.

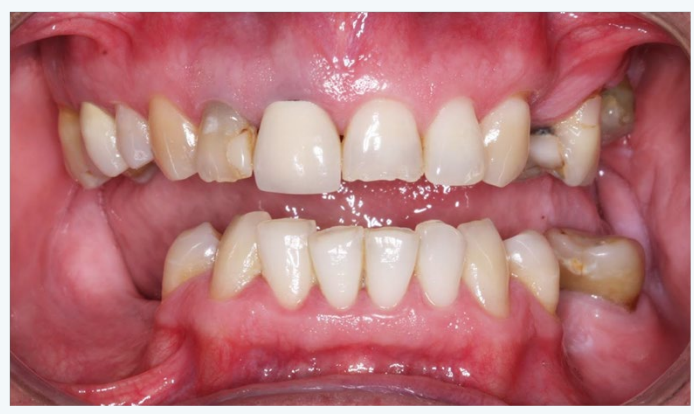

Figure 1B: Preoperative frontal view with partial opening.

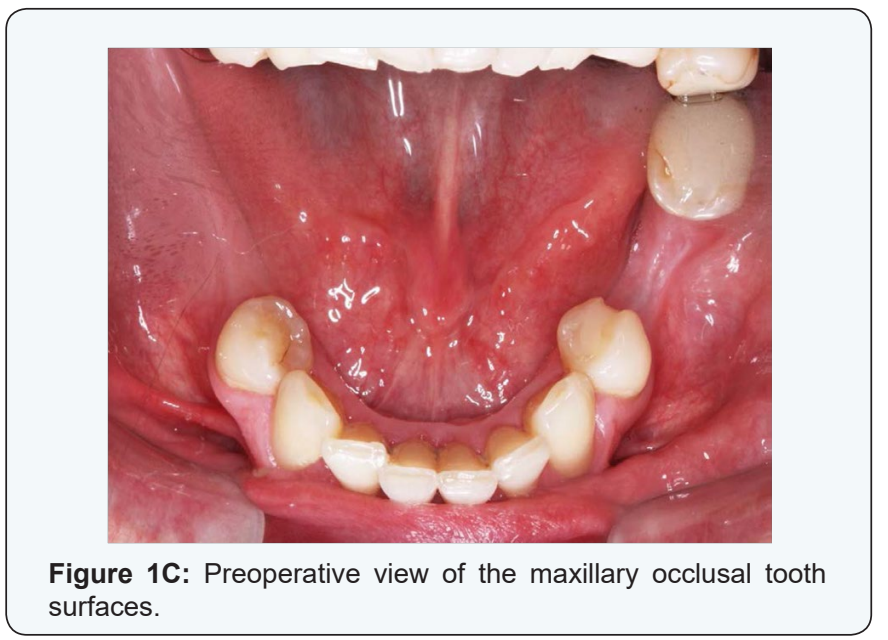

Figure 1C: Preoperative view of the maxillary occlusal tooth surfaces.

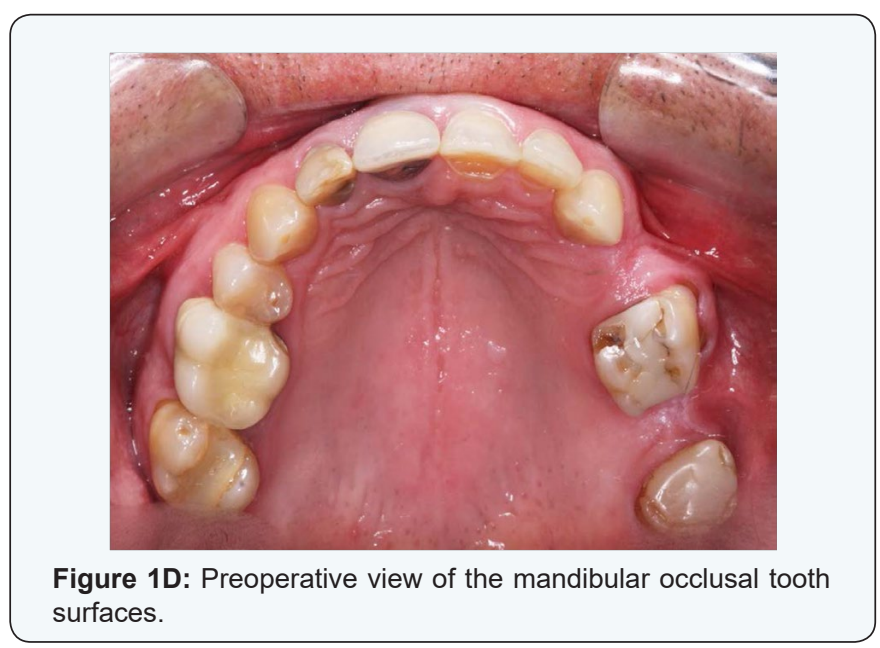




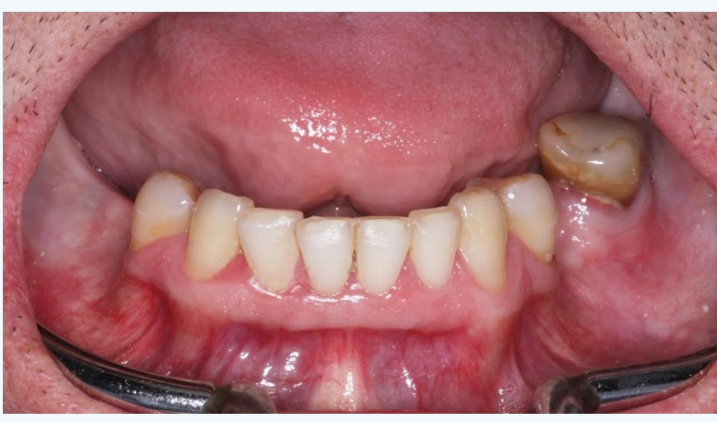

Figure 1E: Preoperative frontal view of the mandibular teeth.

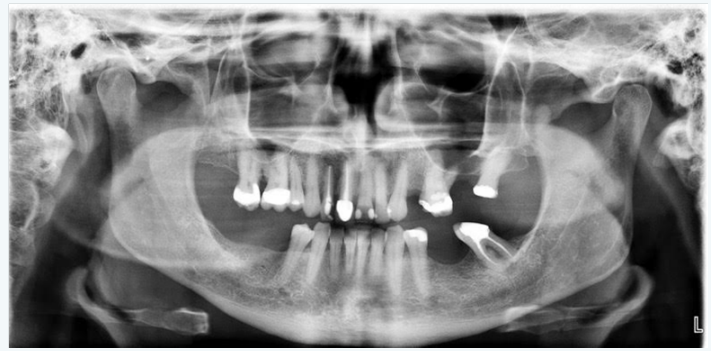

Figure 2: Preoperative panoramic radiograph.

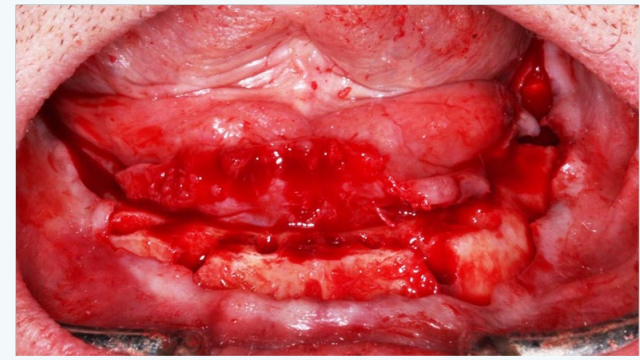

Figure 3B: Mandibular alveloplasty.

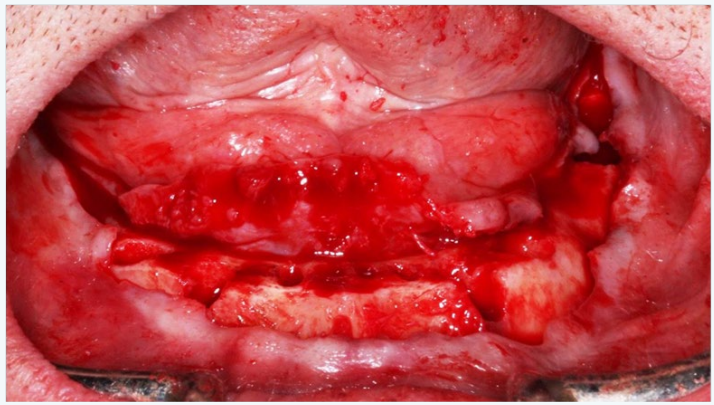

Figure 3B: Mandibular alveloplasty.
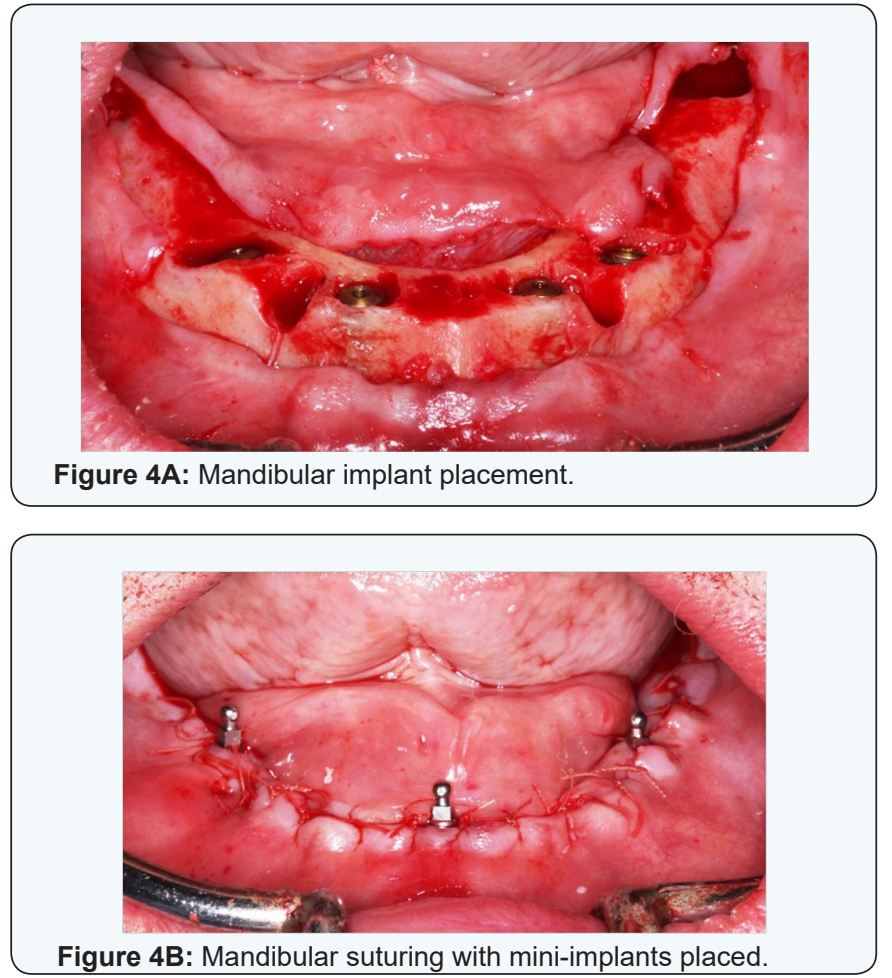

Figure 4B: Mandibular suturing with mini-implants placed
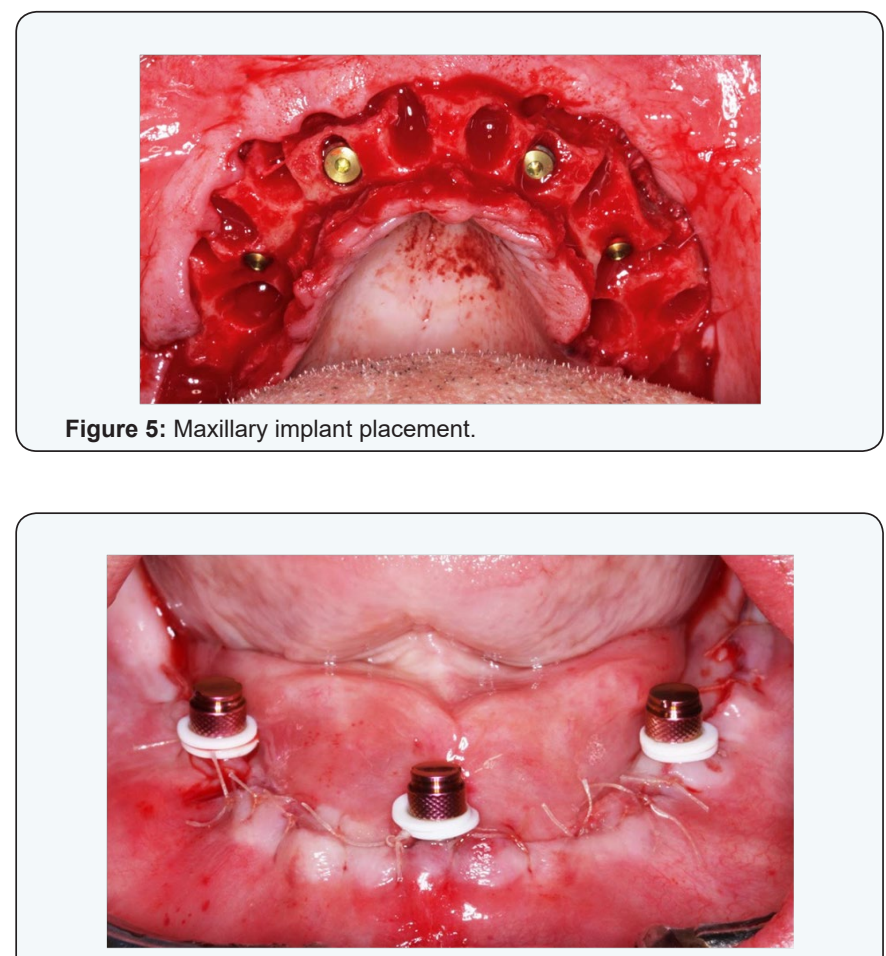

Figure 6A: Mandibular mini-implant retentive housings placed. 

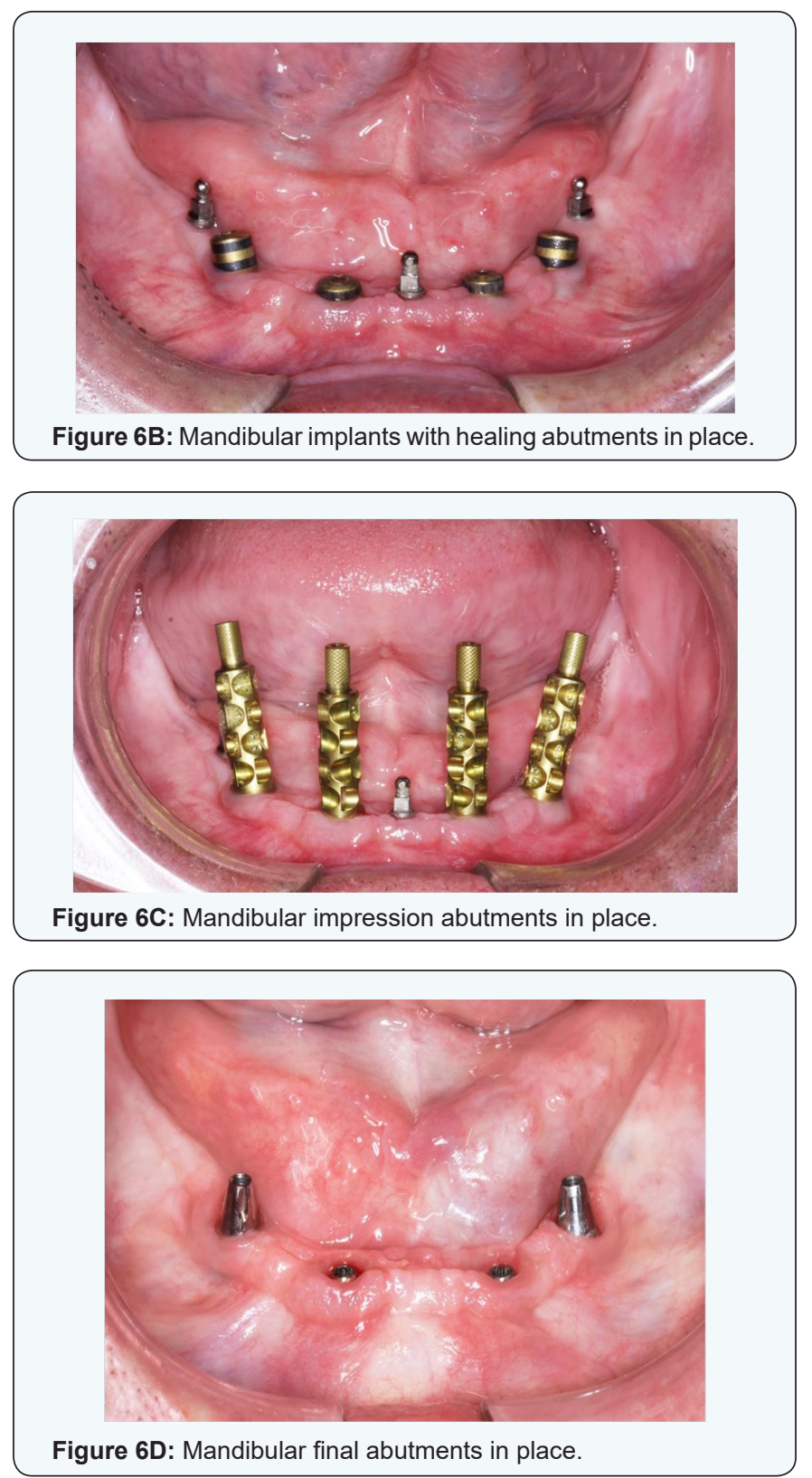

Bone grafting material (Symbios DBX Putty by Dentsply) was placed in the remaining extraction sites and the flaps were approximated without tension and closed with 4-0 PGA sutures (Hu-Friedy). The maxillary transitional denture was then delivered after relining with Coe-Soft. Four weeks later, the patient's mandibular teeth were extracted without complication and alveoloplasty was similarly performed to smooth and level the boney ridge in preparation for implant placement. Care was given to remove sufficient osseous height for his future prosthetic needs, noting that approximately $2.5 \mathrm{~mm}$ of the inter-arch space required was actually gained by opening the patient's occlusion to regain ideal vertical dimension. Following mandibular alveoloplasty, the soft tissue was carefully reflected to visualize the mental foramina, and both posterior implants were sited accordingly. Paralleling pins and digital radiographs were used, as with the maxillary posterior implants, to ensure the enlarging osteotomies would not encroach upon the mental foramina or anterior loops of the mental nerves. The two anterior implants were then sited appropriately between each other. All four implants (ASTRA TECH EV by Dentsply, $4.2 \mathrm{x}$ $13 \mathrm{~mm}$ ) were placed at $45 \mathrm{Ncm}$, following the manufacturer's protocol; sequentially enlarging the osteotomies, and with copious sterile saline irrigation. After utilizing the prime mandibular positions for the permanent implants, sites were then selected for the mini implants and they were placed to a torque value of $40 \mathrm{~N} \cdot \mathrm{cm}$. A $13 \mathrm{~mm}$ implant was placement in the anterior area, while $10 \mathrm{~mm}$ implants were used posteriorly to stay well superior to the mandibular canals. Bone grafting material (Symbios DBX Putty by Dentsply) was placed in the remaining extraction sites and the flaps were approximated without tension and closed with 4-0 PGA sutures (Hu-Friedy). The O-ring housings were placed on the mini implants with white rubber spacers used to prevent subsequent acrylic from locking into undercuts should the implants not be perfectly parallel. The underside of the transitional denture was hollowed out to allow complete seating on the tissue without binding or impinging on the 0-ring housings. Following application of the associated primer, EZ Pickup (Sterngold) was used to capture the housings into the denture and provide tissue adaptation to the newly sutured gingiva. After curing, excess material was then trimmed and polished away, and the transitional denture was inserted with instructions to leave in place for the first 24 hours to help contain any intra-oral swelling. Thereafter, Dale was instructed to remove his lower temporary denture each evening to allow fresh air to better reach the tissue, and also to rinse with Peridex, approximately 2-3 times daily. A total of four months was allowed for maxillary implant integration, with a concurrent three months given to the implants in the mandible. All eight implants were uncovered at a single appointment and healing abutments were placed, with the tissue closed again with 4-0 PGA sutures (Figure 7A\&7B). The maxillary transitional denture was relined with Coe-Soft to accommodate the new abutments, while the lower transitional denture required more preparation. The hard EZ-Pickup that was used to capture the 0-ring housings previously was largely removed with an acrylic burr, while taking care not to loosen the housings themselves (Figure 8A\&8B). CoeSoft was then used to fit the underside of the transitional denture to the new abutments. Peridex use was resumed to help keep the tissue, sutures and abutments clean (Figure 9). One week later all sutures were removed and the patient was instructed to start brushing his tissue and abutments, not just to help keep them clean, but to also stimulate the soft tissue blood flow, and aid in its maturation. At this time initial impressions were made to send to the lab (Root Dental). Another two weeks were allowed for the soft tissue to gain its near-final dimensions prior to final impressions. The healing abutments were removed and open tray impression pins were placed. Custom trays were used with Aquasil Ultra (Dentsply) for final impressions. Tray design for 
implant impressions is critical, and must have sufficient space in the implant region to allow impression material to completely encase the impression pins (Figure 10A\&10B). Otherwise, the pins can "flex" in the elastomeric impression material and affect the accuracy of not just the final model, but the framework and subsequent final prosthesis as well. Once final models were fabricated with the appropriate implant abutments, conventional baseplates with wax rims were made and fitted. A subsequent teeth-in-wax try-in was performed to establish ideal vertical dimension and facial form, ideal teeth and smile esthetics, and ideal phonetics. After approval by the patient, the case was returned to Root Lab where final Astra abutments were selected the frameworks were made. The maxillary and mandibular frameworks and their associated abutments were tried in and the fit verified visually, tactilely and radiographically prior to returning to the lab for final processing (Figure 11A\&11B). At the final delivery, the mini implants were easily removed (unthreaded, without the need for local anesthetic) and the final abutments placed, and hybrids delivered, with all components torqued to Astra's specifications (Figure 12). The screw access openings were sealed with silicone tape and tooth-colored or gingival colored composite resin (Herculite Ultra Flow by Kerr) as the site called for. Final occlusal balancing was negligible and easily performed to provide Dale the potential for bilateral group function occlusion (Figure 13A\&13B). Optimal hygiene was discussed and demonstrated using a Sonicare, Water Pik, floss threaders or Oral-B Superfloss.
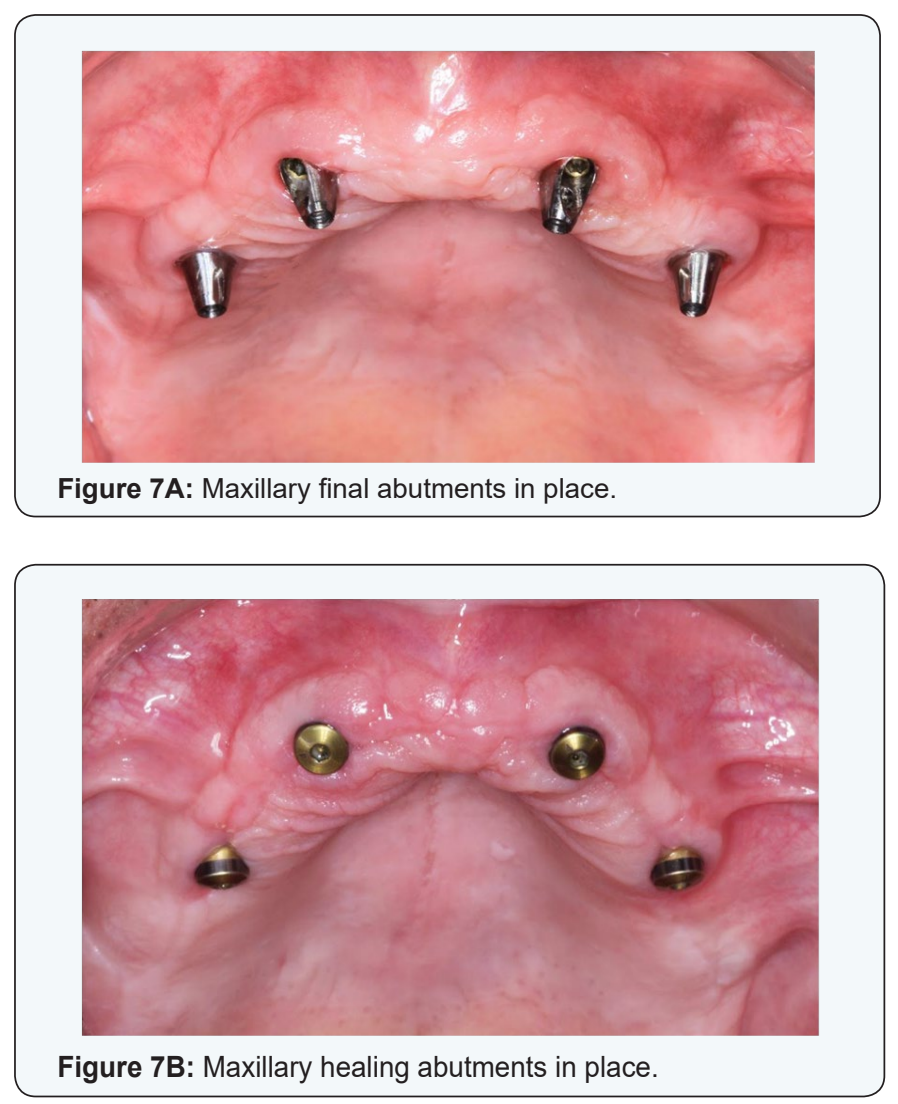

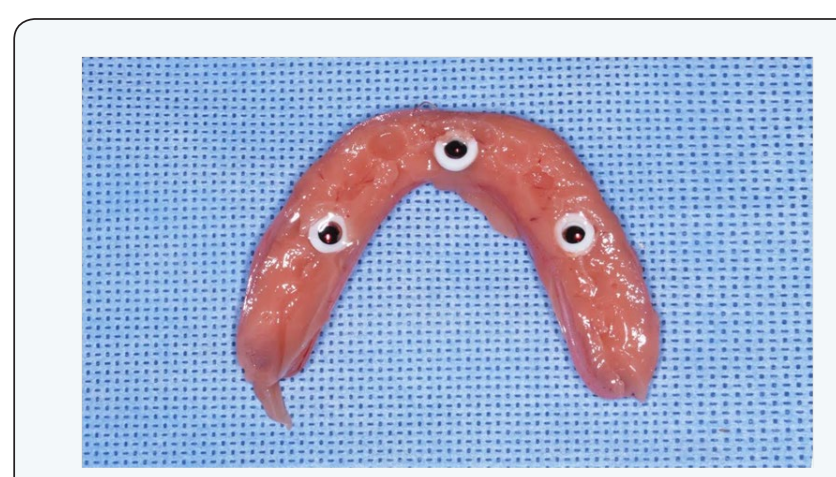

Figure 8A: Mandibular intaglio surface of transitional denture immediately after picking up retentive housings.
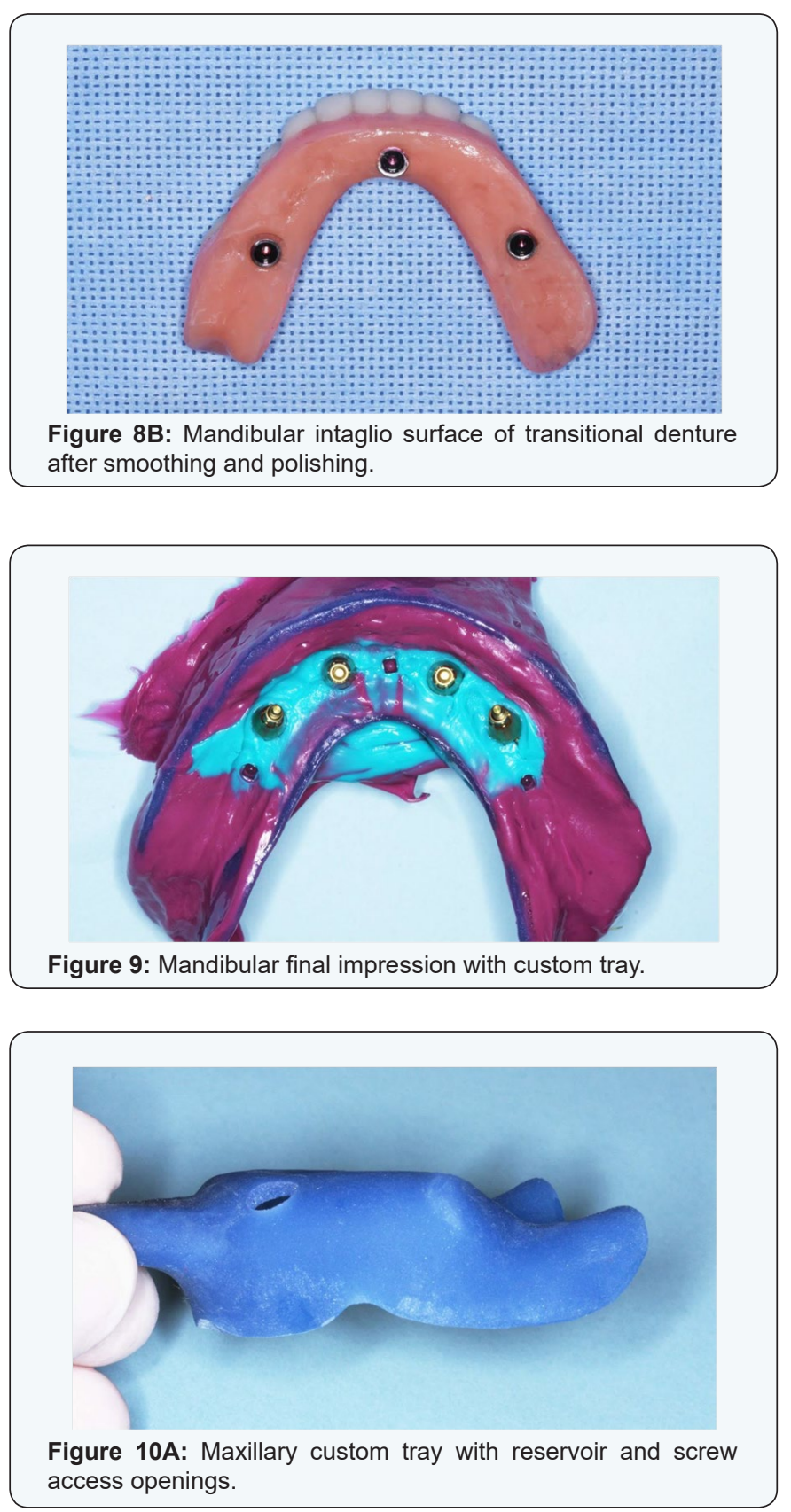


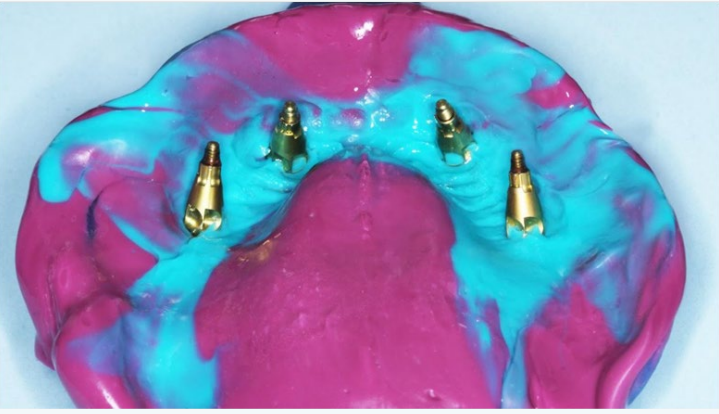

Figure 10B: Maxillary final impression with custom tray.

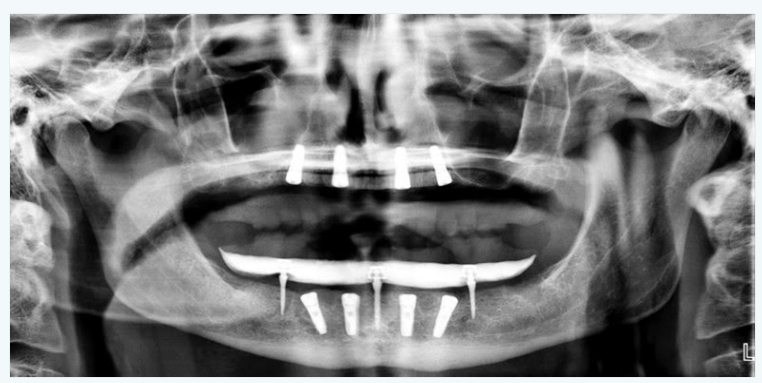

Figure 11A: Mid-procedure implant placement panoramic radiographic.

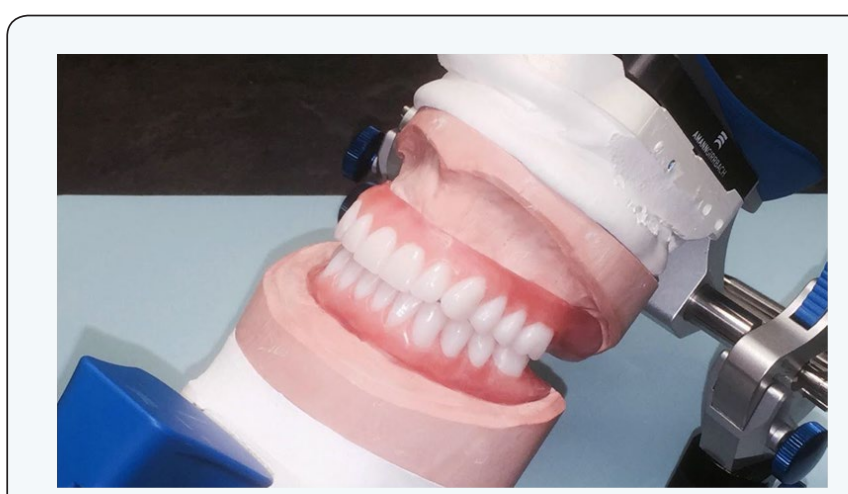

Figure 12: Teeth in wax set-up on articulator.

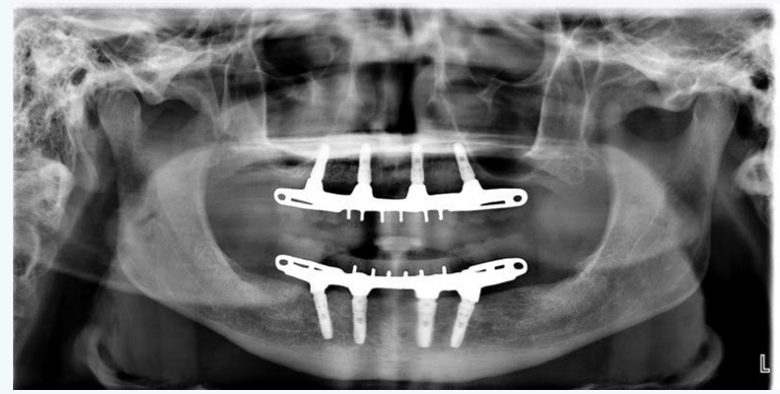

Figure 11B: Final panoramic radiograph of implants with hybrid bridges in place.
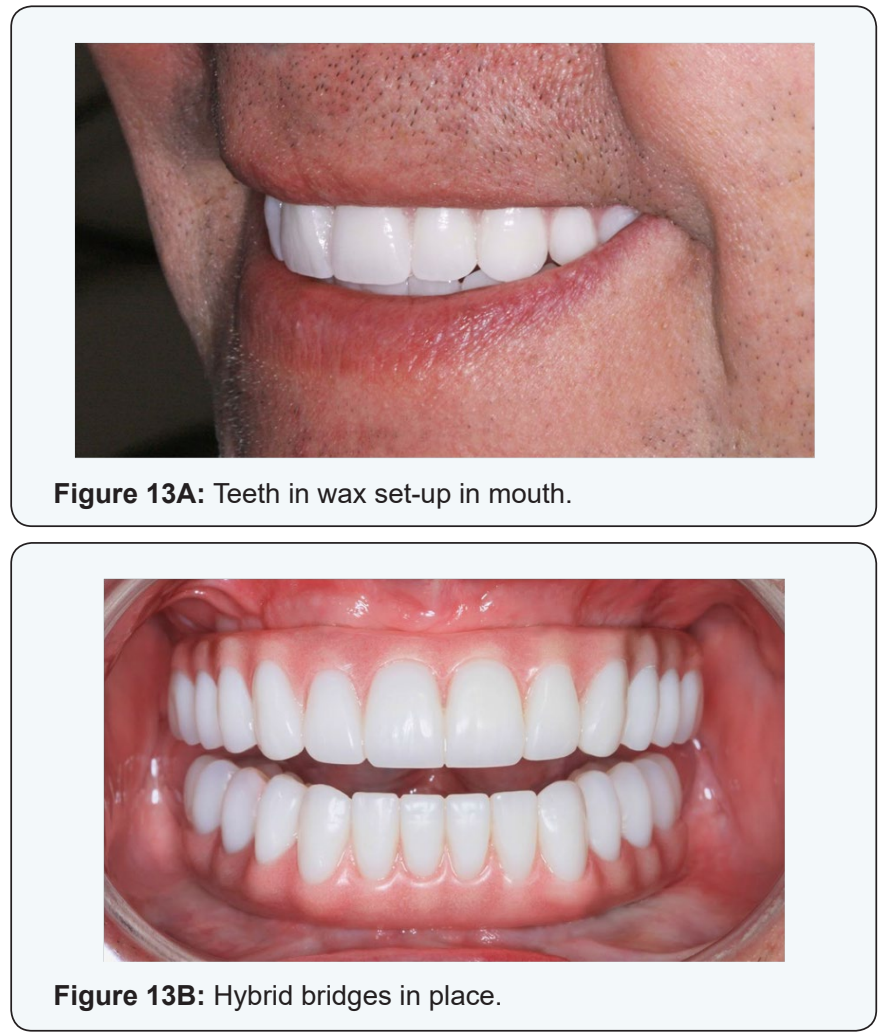

\section{Case 2}

Joe is a 53 year old male who presented to our office saying he had already been told he needed to have his remaining teeth removed and additional implants placed in his maxillary arch, along with implants added in his mandibular arch, to support full arch Zirconia bridges. His natural teeth and surrounding bone suffered from severe periodontal disease, but the patient needed to be informed that his three maxillary left implants suffered from extensive bone loss. This was initially seen on his digital panoramic radiograph, with the severe extent of the periimplantitis confirmed with digital periapical radiographs (Figure 14). The patient was surprised to learn that bone can be lost around implants and desired to understand the conditions and situations causing this loss [11]. Because of the uncertain and numerous potential factors that could cause bone loss, the primary supposition was that a continuation of a local or systemic process may have led to the bone loss around his natural teeth. Additionally, an associative factor for this patient was the fixed prosthesis design attached to the implants, and how it affected the peculiar type of occlusion [12]. The patient has a skeletal Class III maxilla-mandibular relationship, with an additional skeletal posterior bilateral crossbite tendency (Figure 15A-15D). His maxillary alveolar osseous structure is significantly smaller in every dimension than that of his mandible. But, the patient is dentally compensated to such a degree that all maxillary teeth 
flare significantly toward the facial to "fit over" his mandibular teeth. Consequently, when his maxillary left implants were inserted, they were placed in alveolar bone that was substantially palatal to the opposing mandibular teeth. In addition, the patient's subsequent screw-retained $10 \times 12$ × 14 porcelainfused-to-metal (PFM) fixed prosthesis was designed to flare facially as his natural teeth had presented. This had the unfortunate side effect of creating severe non-vertical, off-axes forces on his implants and surrounding bone. This condition accounted for the significant bone loss. Additionally, in an effort to visually mask the flaring of the prosthetic teeth, the original lab technician created a large facial "ridge-lap" of pink porcelain. This had the unfortunate side effect of creating a significant food and bacterial trap that contributed to the bone loss around the supporting implants. As a result of these contributing factors, it was not recommended that his three current implants be considered healthy enough to help support a final restoration. The option of removing these implants was discussed and grafting the sites with the hope that they specifically, or the region in general, would support new implants in the future. This would result in significant loss of surrounding alveolar bone, and would likely entail 4 - 6 months of graft maturation, with no guarantee of success. It was conceivable that the bone grafting might prove less than successful, with the potential loss of more alveolar structure. If this was to occur, a conventional sinus lift would prove necessary. The patient desired not to wear a transitional denture for an extensive period of time, especially without the assurance that implant placement would be located in preferred areas. Therefore, the implants were opted to be put "to sleep." This was expected to serve dual purposes: 1) allow the placement of new implants immediately in the previously nonimplanted adjacent sites, and 2) allow the submerged implants that were put "to sleep" to serve as a buttress for the bone. A lengthy discussion ensued with the patient regarding the design and type of final prostheses that would be appropriate. The possibility of screw-retained Hybrid bridges, specifically made in Zirconia, was rejected because his alveolar arch form discrepancy (underbite and crossbite) fixed prostheses would prove very problematic. The resulting smile esthetics and facial form of this type of prosthesis would be untenable. Although Zirconia might be considered a "strong" material, it has no functional resilience. In the incorrect occlusal scheme (compensated underbite/crossbite), this could transmit significantly more force to the underlying implants, thus essentially ensuring their failure from simple mechanical overload. An acrylic hybrid bridge might prove more resilient, and thus be potentially "kinder" to the underlying implants, but in a similarly inappropriate occlusal scheme it would be expected to fracture readily (most likely in the anterior region). Finally, a PFM hybrid might be considered stronger than acrylic, but porcelain has little shear strength, and in a flared design attempting to compensate for an underbite/crossbite fractures would be considered the expectation, rather than the exception
Ideal esthetics were paramount to the patient. Optimal and easy hygiene were similarly paramount. Protecting the new implants and their overlying prostheses from inappropriate and damaging forces was critical. Ease of repair or replacement when away from home was not considered a luxury. Consequently, the recommendation was given for removable over-dentures, rather than screw-retained hybrid bridges. The patient had to overcome the predisposed bias that "removable" teeth were somehow inferior to "permanent" teeth. A wide-variety of patients and needs can benefit from different types of prostheses, and for different reasons. As such, removable prostheses could solve the patient's problems by optimally enhancing oral hygiene. The prostheses can be removed from the mouth for unimpeded access to the implants, abutments and surrounding tissue. Also from an esthetic standpoint, the patient desired a natural facial appearance, and over-dentures allows a facial flange on the upper prosthesis with sufficient fullness to provide the right balance to the profile. Treatment was initiated by removing the patient's remaining maxillary teeth and performing sufficient alveoloplasty to smooth and level the remaining alveolar ridge (Figure 16A\&16B). Visual confirmation of the anticipated radiographic profile regarding the three maxillary left implants occurred, exposed portions of the fixtures were removed, the new coronal surfaces were smoothed, and the internal portions were filled with composite resin. Six new implants (ASTRA TECH TX by Dentsply, $4.5 \times 11 \mathrm{~mm}$ in all positions, except the maxillary left posterior, which was $4.5 \times 9.0$ ) were placed evenly throughout the arch. All implants were placed at $40 \mathrm{~N} \bullet \mathrm{cm}$, following the manufacturer's protocol of sequentially enlarging the osteotomies, and using copious sterile saline irrigation. Bone grafting material (Symbios DBX Putty by Dentslpy) was placed in the remaining extraction sites and the flaps were approximated without tension and closed with 4-0 PGA sutures (Hu-Friedy). The maxillary transitional denture was then delivered after relining with Coe-Soft. It's worth noting that the maxillary transitional denture was designed with approximately $2 \mathrm{~mm}$ of vertical dimension opening to help compensate for the patient's shifting and over-closure. This increased vertical dimension also had the effect of rotating the mandible posteriorly and inferiorly, thus lessening his Class III facial appearance. Four weeks later, the patient's mandibular teeth were extracted without complication and judicious alveoloplasty was similarly performed to smooth and level the osseous ridge in preparation for implant placement (Figure 17A\&17B). Care was given to removing sufficient osseous height for his future prosthetic needs. Six new implants (ASTRA TECH TX by Dentsply, 4.5 x 11.0 in the four anterior positions, and $5.0 \times 9.0$ in the two posterior positions) were placed evenly spaced throughout the arch (Figure 18A-18C). All implants were placed at $40 \mathrm{~N} \bullet \mathrm{cm}$, following the manufacturer's protocol of sequentially enlarging the osteotomies, and using copious sterile saline irrigation. The anterior implants were placed with a slight lingual angulation to allow the new prosthetic teeth to be placed as far lingually as 
possible, and to help minimize the patient's previous Class III facial appearance. Bone grafting material (Symbios DBX Putty by Dentslpy) was placed in the remaining extraction sites and the flaps were approximated without tension and closed with 4-0 PGA sutures (Hu-Friedy). The maxillary transitional denture was then delivered after relining with Coe-Soft. After utilizing the prime mandibular positions for the permanent implants, sites were then selected for the mini implants (MDI by 3M, $1.8 \times 10.0$ $\mathrm{mm}$ ), which were placed to a torque value of $40 \mathrm{~N} \bullet \mathrm{cm}$. Bone grafting material (Symbios DBX Putty by Dentsply) was inserted into the remaining extraction sites and the flaps were approximated without tension and closed with 4-0 PGA sutures (Hu-Friedy). The O-ring housings were placed on the mini implants with white rubber spacers used to prevent subsequent acrylic from locking into undercuts, should the implants not be perfectly parallel. The underside of the transitional denture was hollowed out to allow complete seating on the tissue without binding or impinging on the O-ring housings. Following application of the associated primer, EZ Pickup (Sterngold) was used to capture the housings into the denture and provide tissue adaptation to the newly sutured gingiva. After curing, excess material was then trimmed and polished away, and the transitional denture was inserted with instructions to leave in place for the first 24 hours to help contain any intra-oral swelling. Thereafter, the patient was instructed to remove his mandibular temporary denture every evening to allow fresh air to better reach the tissue, and also to rinse with Peridex approximately 2-3 times daily. Three months was allotted for maxillary implant integration, with a concurrent two months given to the mandibular implants. All twelve implants were uncovered at a single appointment and healing abutments were placed, with the tissue closed again with 4-0 PGA sutures (Figure 19A-19D). The maxillary transitional denture was readily relined with Coe-Soft to accommodate the new abutments, while the mandibular transitional denture required more preparation (Figure 20A-20C). The hard EZ-Pickup that was used to capture the O-ring housings was removed with an acrylic burr, while care was taken not to loosen the housings. Coe-Soft was used to fit the underside of the transitional denture to the new abutments. Peridex use was resumed to help keep the tissue, sutures and abutments clean (Figure 21A-21D). One week later all sutures were removed. The patient was instructed to start brushing the tissue and abutments, for cleanliness purposes and to stimulate the soft tissue blood flow aiding in maturation. Initial impressions were taken, sent to the laboratory (Root Dental). Two weeks were allowed for the soft tissue to gain its near-final dimensions prior to final impressions. The healing abutments were removed and open tray impression pins were placed. Custom trays were used with Aquasil Ultra (Dentsply) for final impressions. Tray design for implant impressions is critical for success, and must have sufficient space in the implant region to allow impression material to completely encase the impression pins. If not, the pins can "flex" in the elastomeric impression material and affect the accuracy of not just the final model, but the framework and subsequent final prosthesis as well. Once final models were fabricated with the appropriate implant abutments, conventional baseplates with wax rims were made and fitted. A subsequent teeth-in-wax try-in was performed to establish ideal vertical dimension and facial form, ideal teeth and smile esthetics, and ideal phonetics. The case was returned to Root Lab where final LOCATOR abutments were selected, the frameworks were made and the final prostheses were processed. An exact duplicate maxillary prosthesis made for the patient to keep, especially for travel purposes. At the final delivery, the mini implants were removed (unthreaded, without local anesthetic) and the final LOCATOR abutments placed, and over-dentures delivered, with all components torqued to Astra's specifications (Figure 22A\&22B). Final occlusal balancing was negligible with all three prostheses (the two maxillary and one mandibular) and easily performed to provide Joe with acceptable bilateral group function occlusion (Figure 23A-23E). Optimal hygiene was discussed and demonstrated using a Sonicare (Figure 24A-24D).

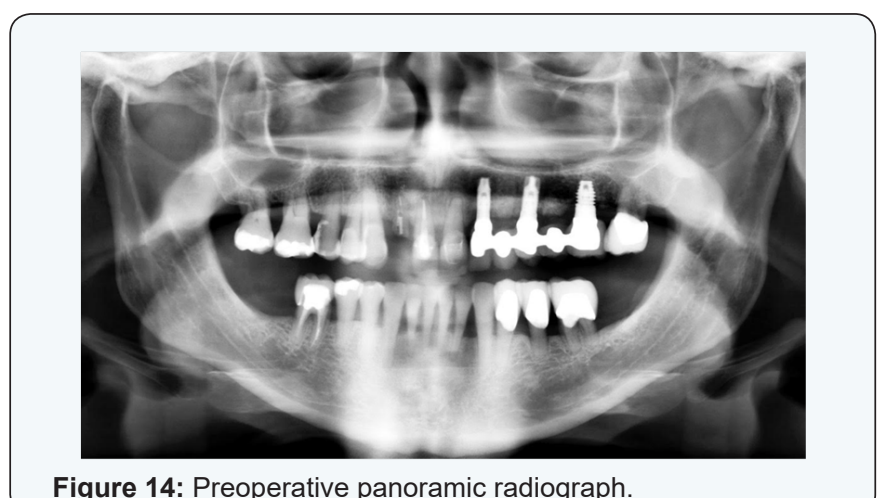

Figure 14: Preoperative panoramic radiograph.

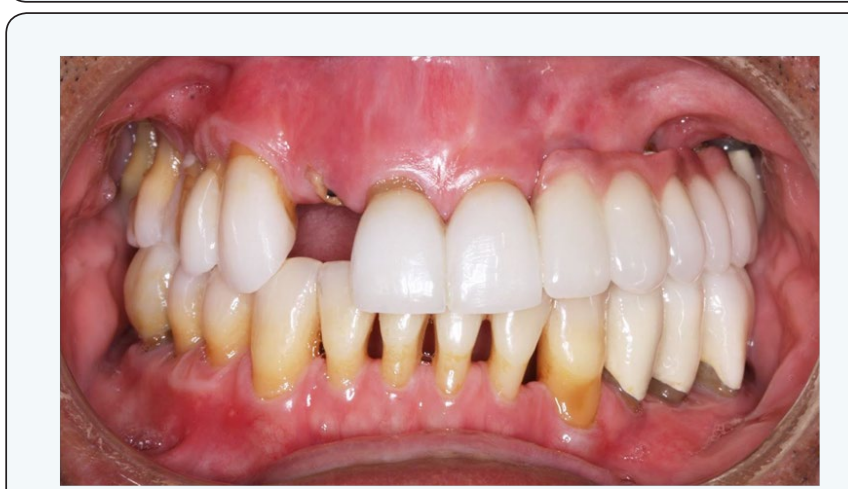

Figure 15A: Preoperative frontal view in maximum intercuspation.

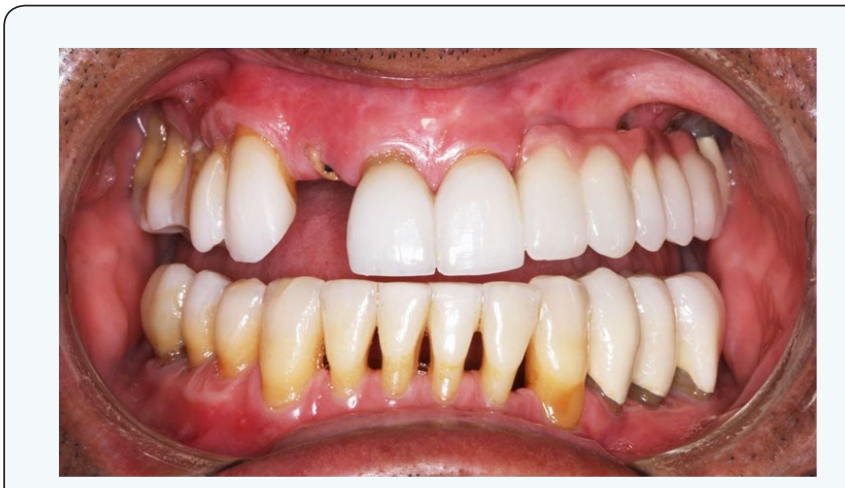

Figure 15B: Preoperative frontal view with mouth open. 


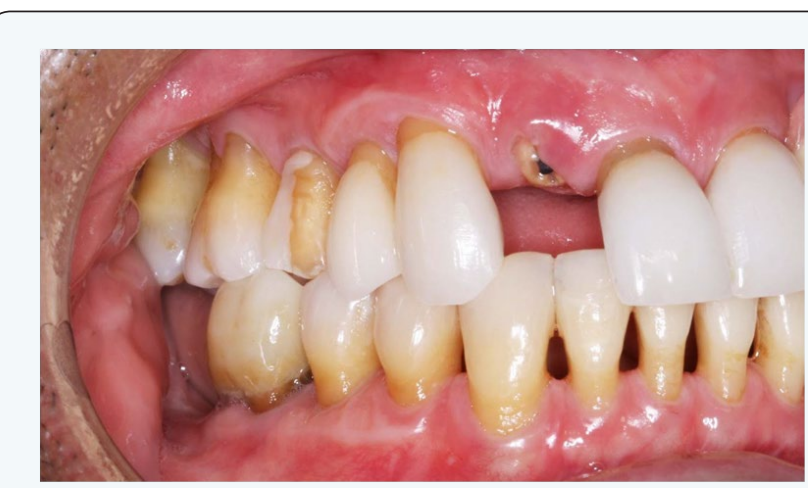

Figure 15C: Preoperative frontal view of anterior-posterior right side.
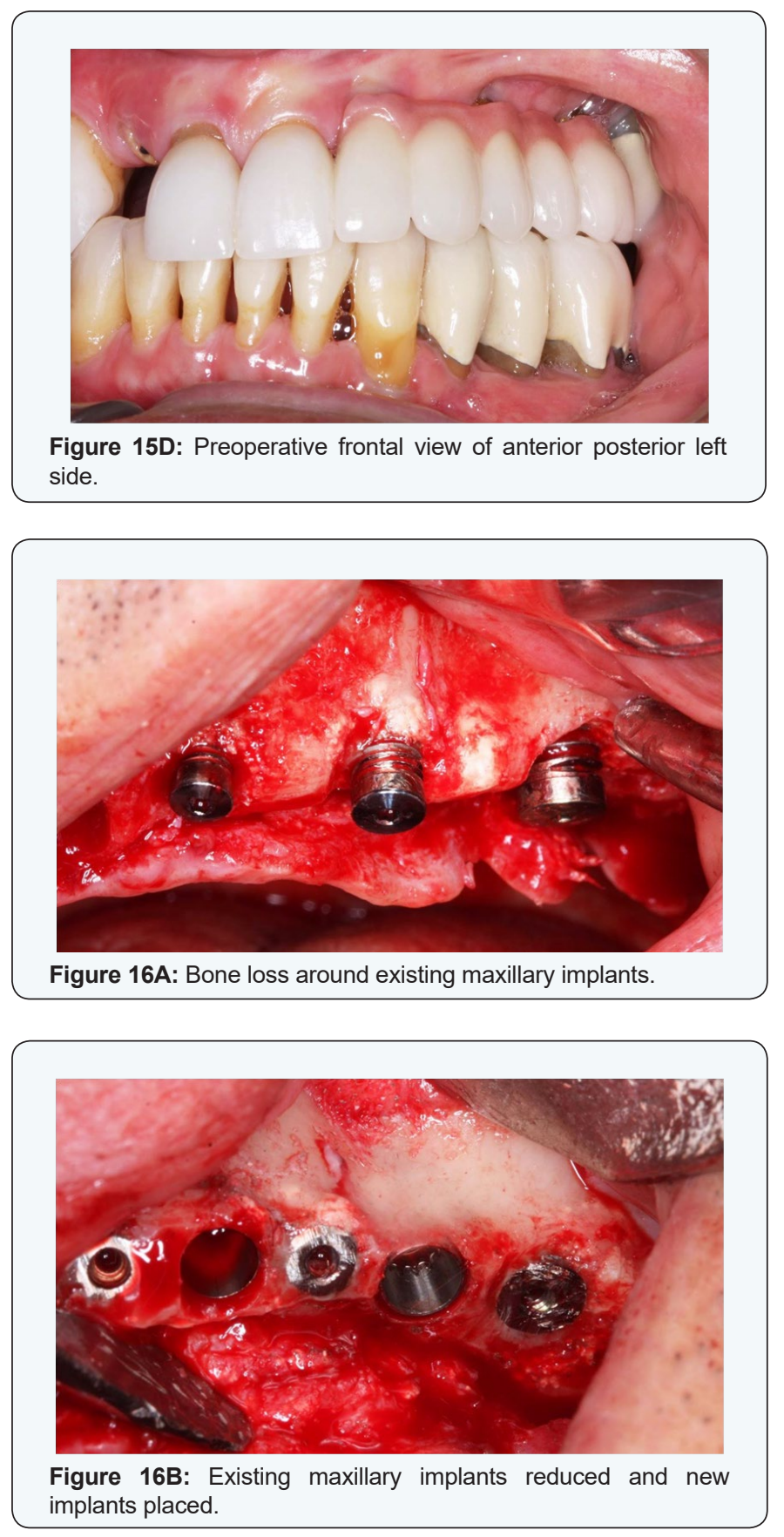

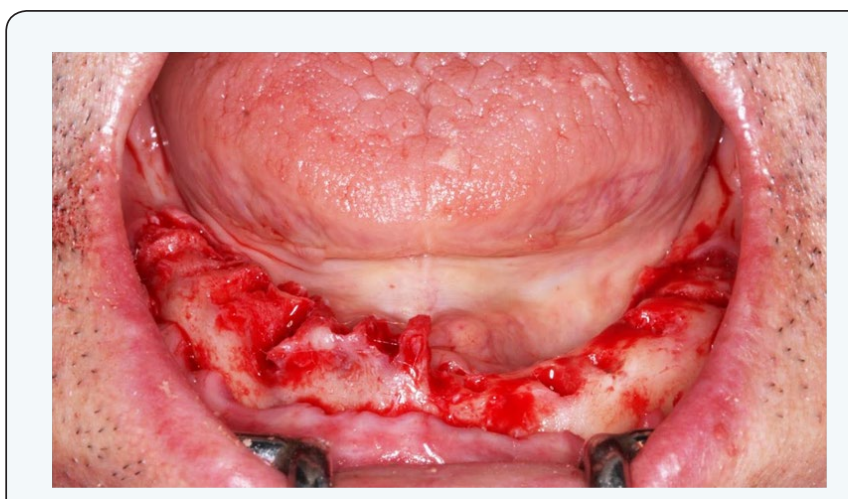

Figure 17A: Mandibular alveoloplasty patient's left side only.

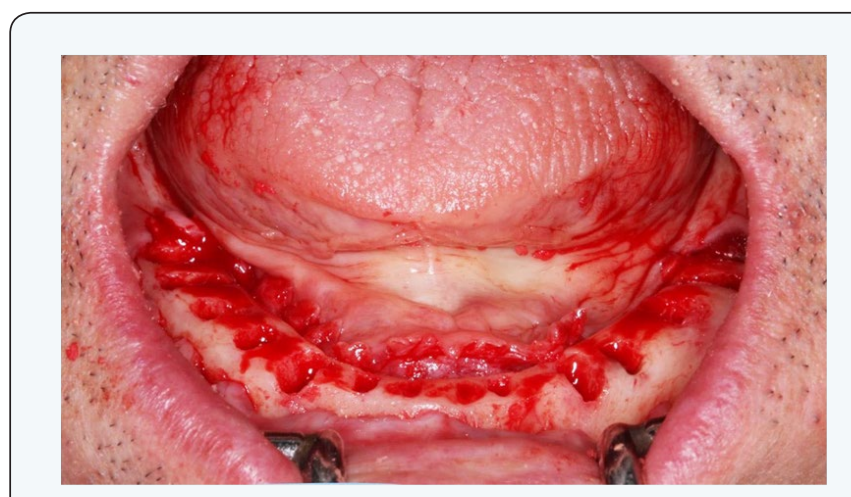

Figure 17B: Mandibular alveoloplasty full arch.
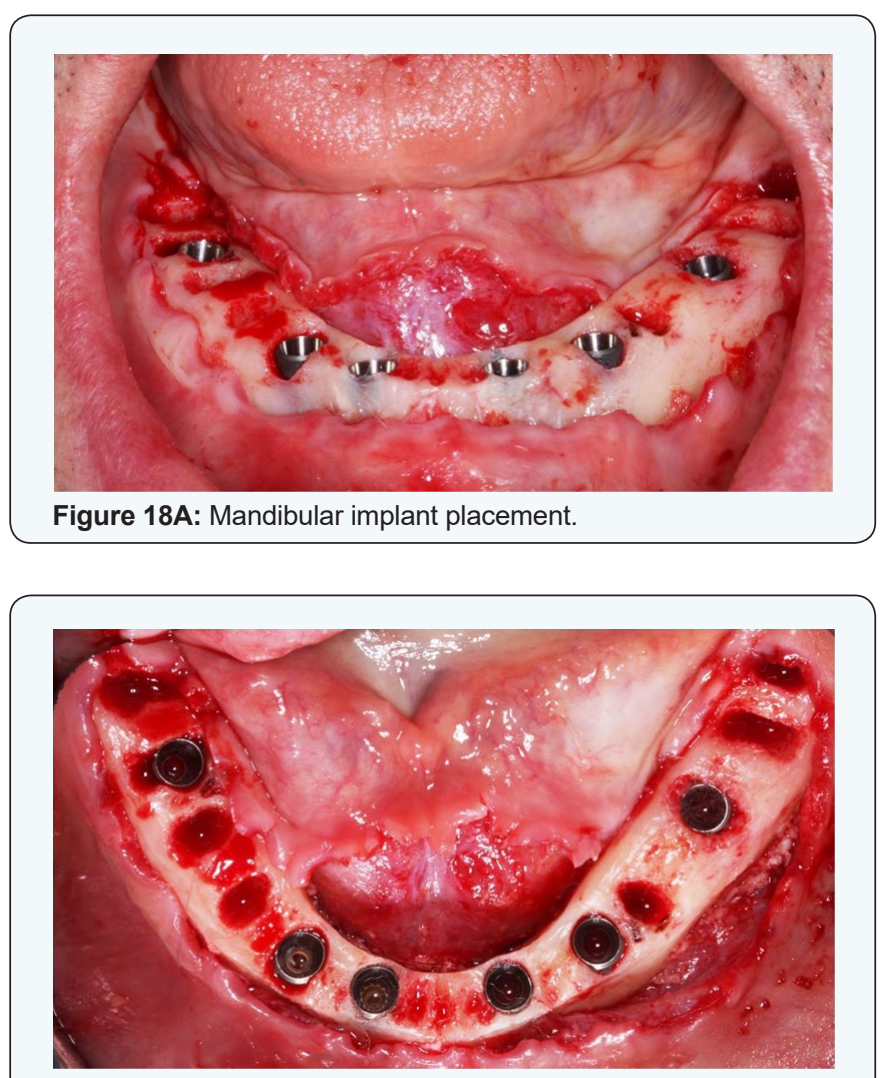

Figure 18B: Mandibular implant placements occlusal view. 


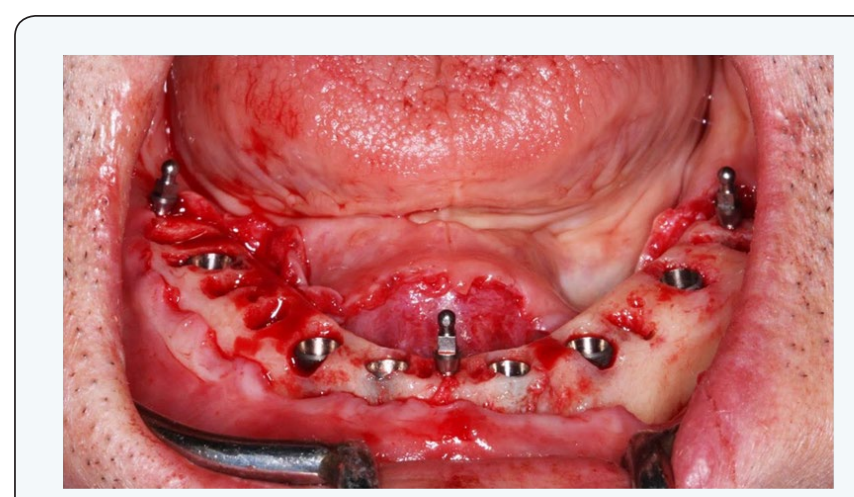

Figure 18C: Mandibular mini implants placed.

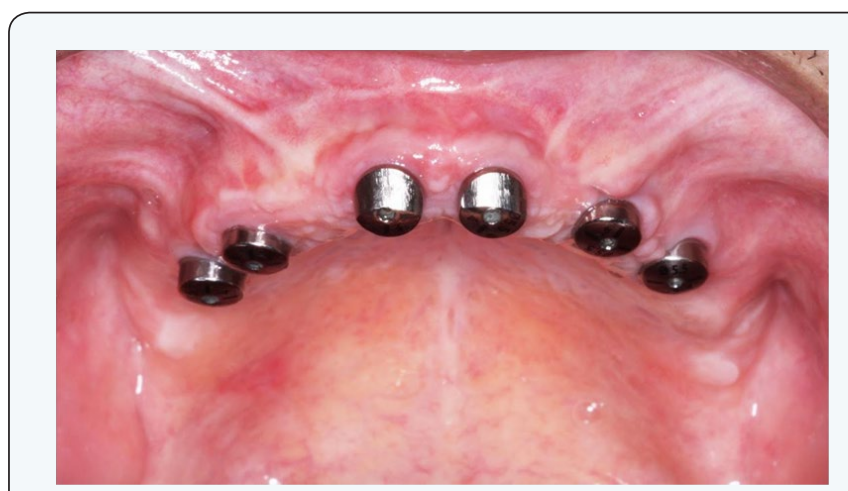

Figure 19A: Maxillary healing abutments.

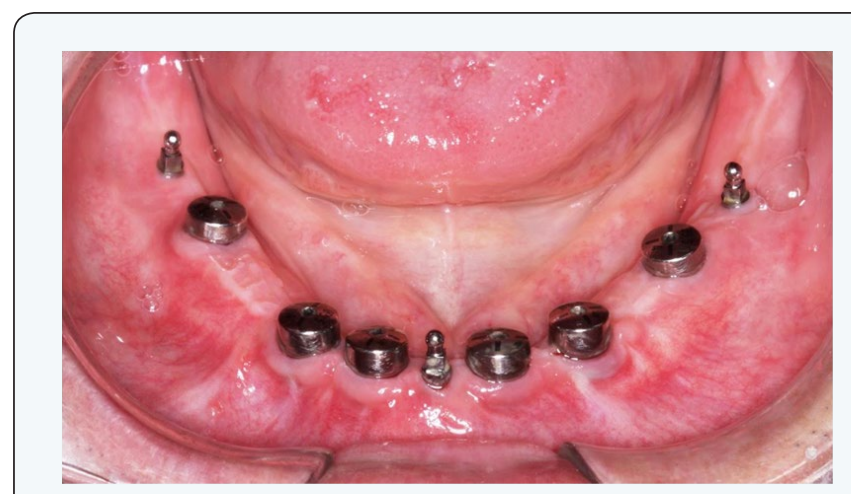

Figure 19B: Mandibular healing abutments.

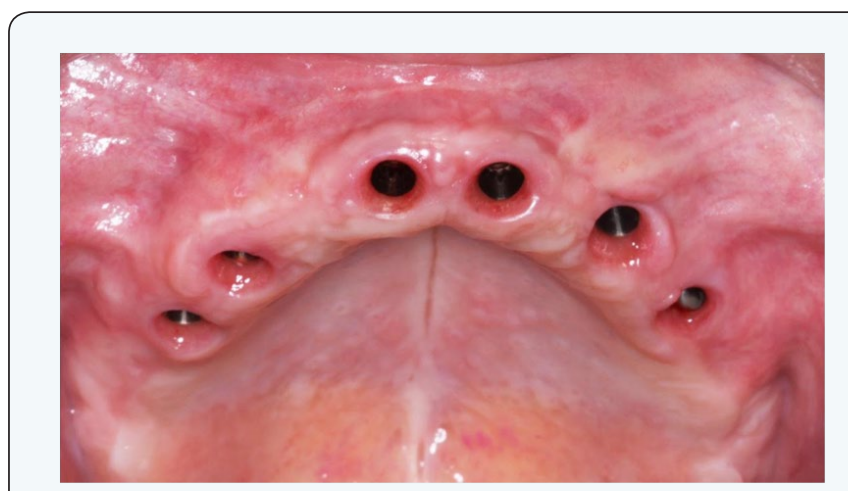

Figure 19C: Maxillary implants after removal of healing abutments.

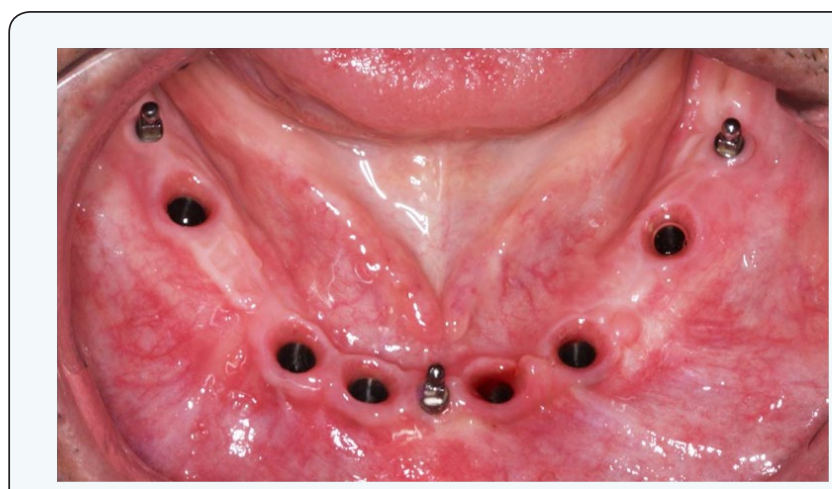

Figure 19D: Mandibular implants after removal of healing abutments.

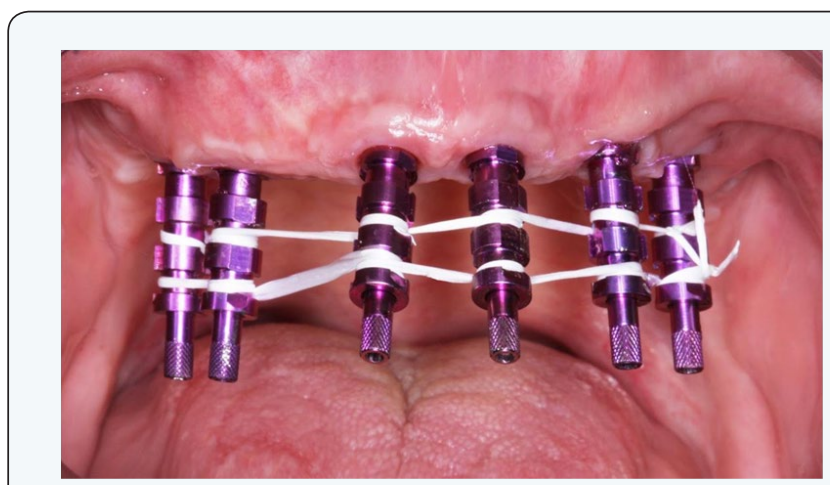

Figure 20A: Maxillary implants with impression abutments placed and floss scaffold.

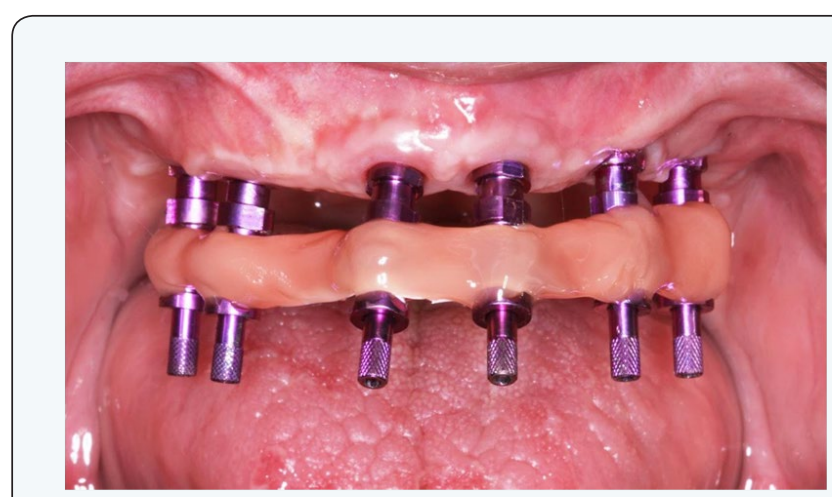

Figure 20B: Maxillary implants with floss scaffold encased in ezpickup.

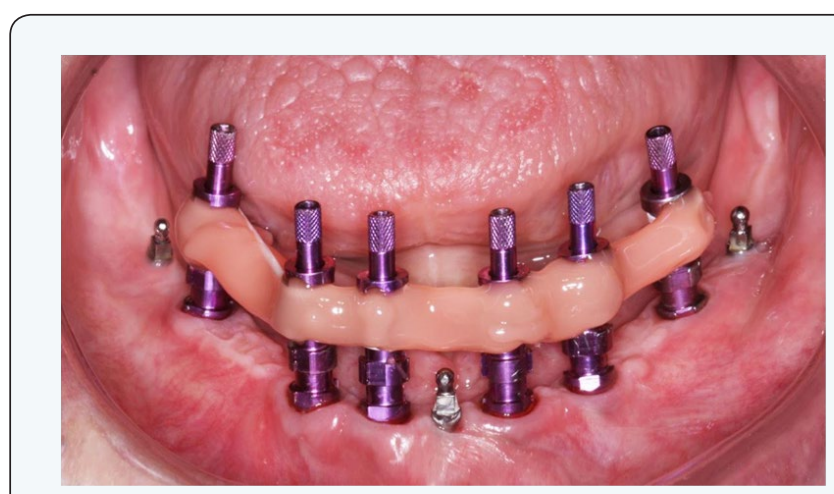

Figure 20C: Mandibular implants with floss scaffold encased in ez-pickup. 


\section{Advances in Dentistry \& Oral Health}

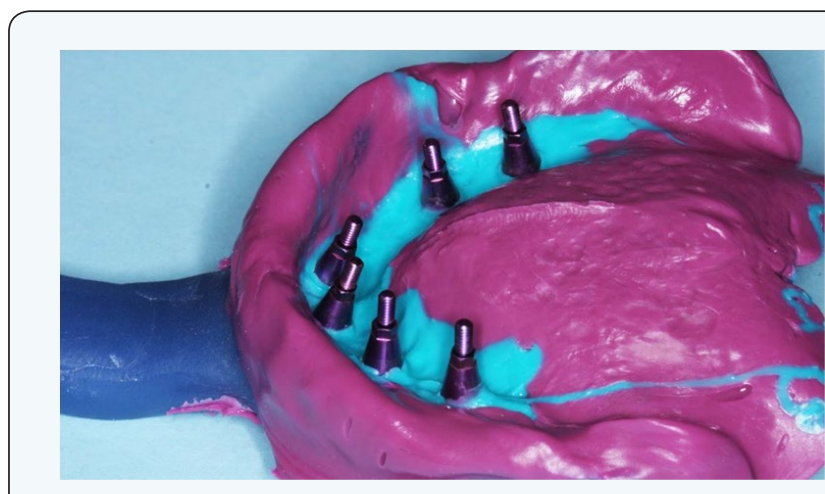

Figure 21A: Maxillary impression with custom tray and impression abutments.

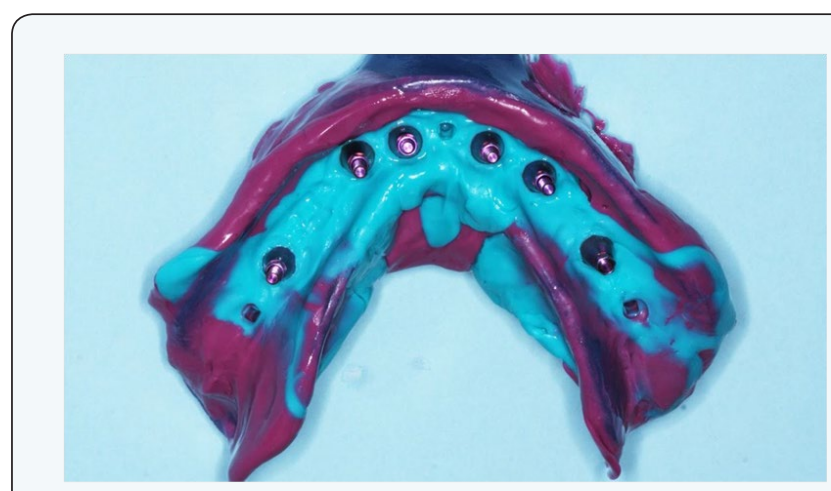

Figure 21B: Mandibular impression with custom tray and impression abutments.

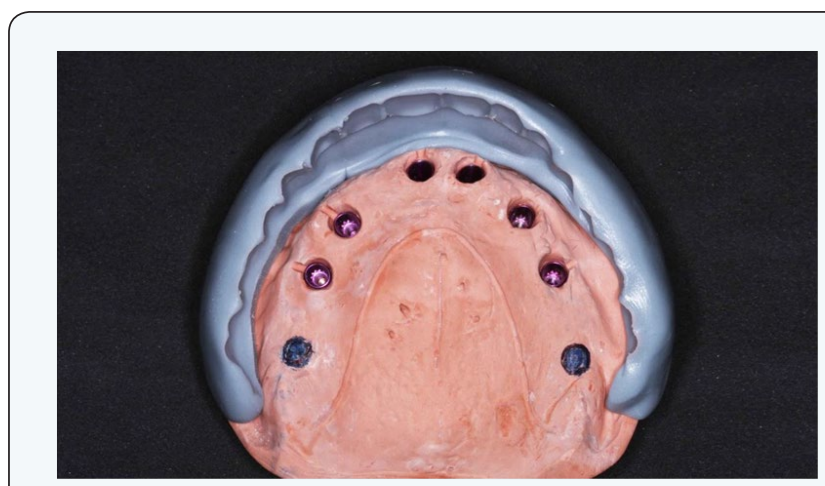

Figure 21C: Maxillary model with sil-tech matrix frontal view.

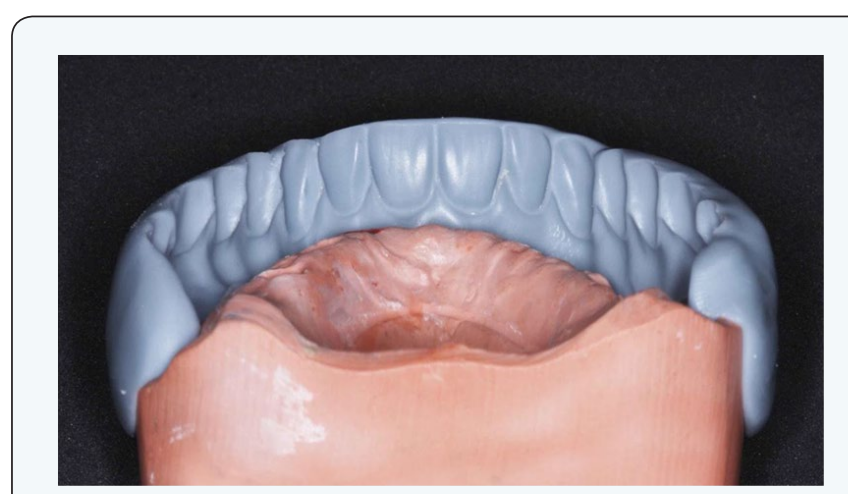

Figure 21D: Maxillary model with sil-tech matrix rear view.

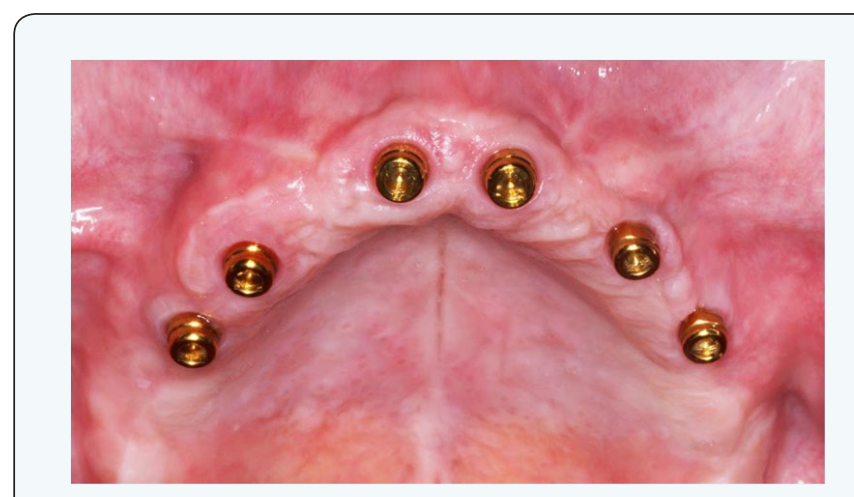

Figure 22A: Maxillary implants with locator abutments.

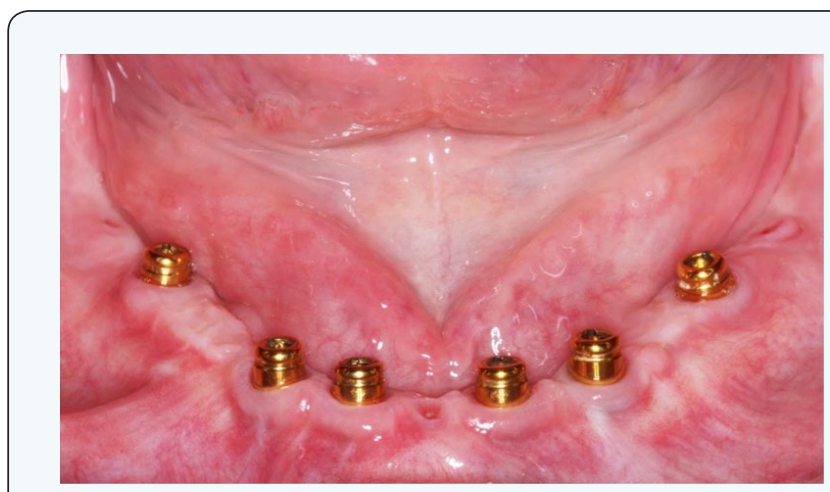

Figure 22B: Mandibular implants with locator abutments.

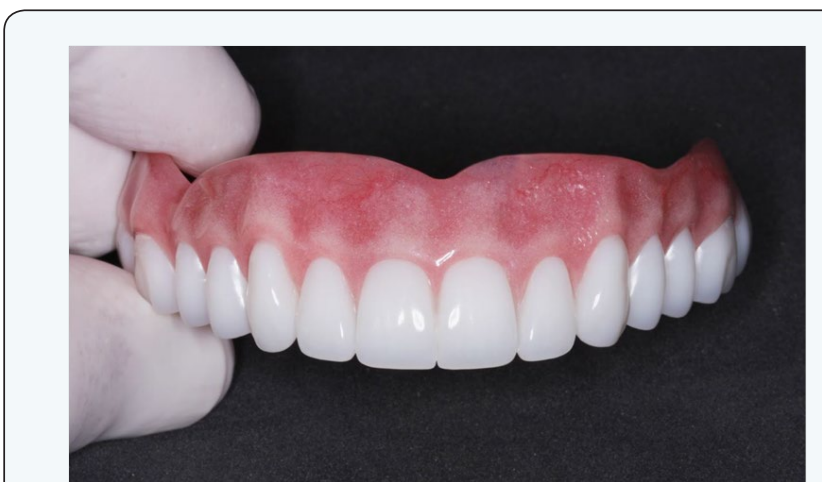

Figure 23A: Maxillary over-denture.

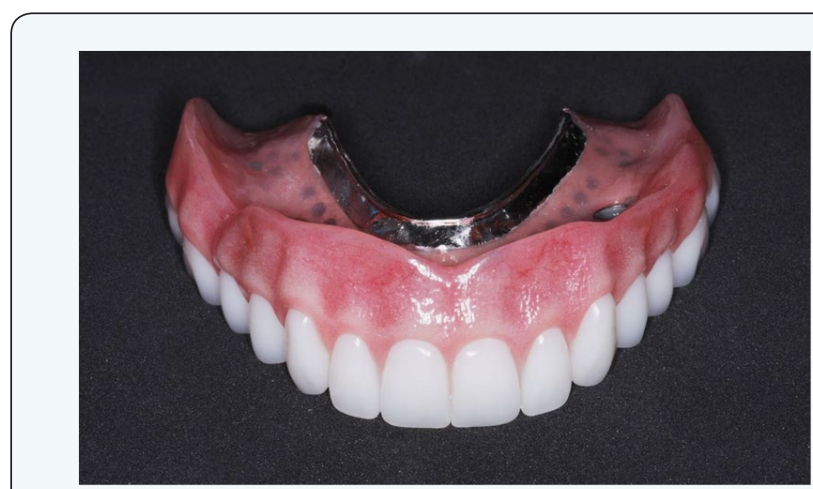

Figure 23B: Maxillary over-denture. 


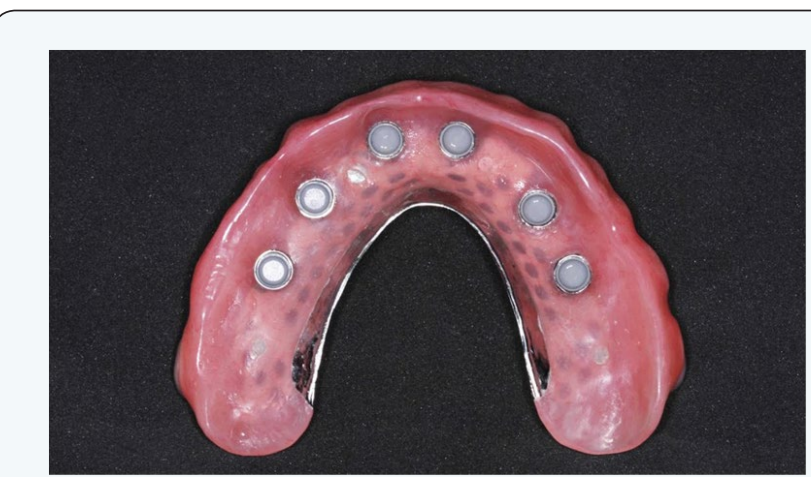

Figure 23C: Intaglio surface of maxillary over-denture.

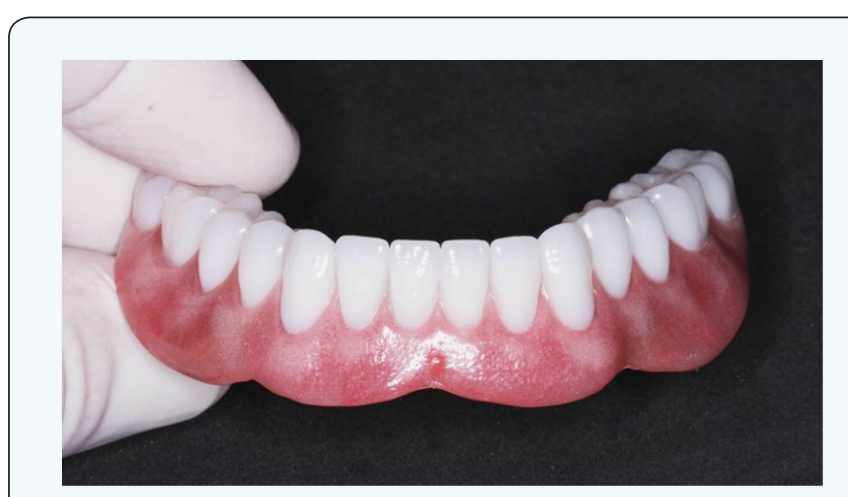

Figure 23D: Mandibular over-denture.

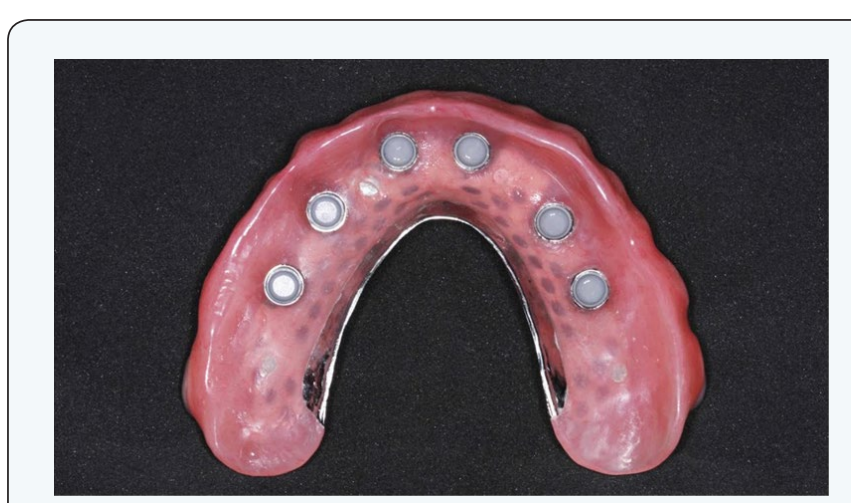

Figure 23C: Intaglio surface of maxillary over-denture.

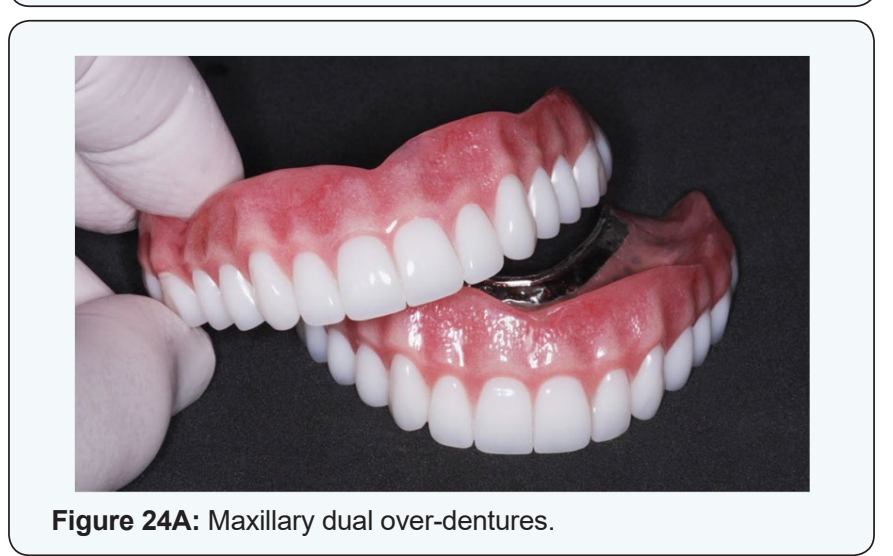

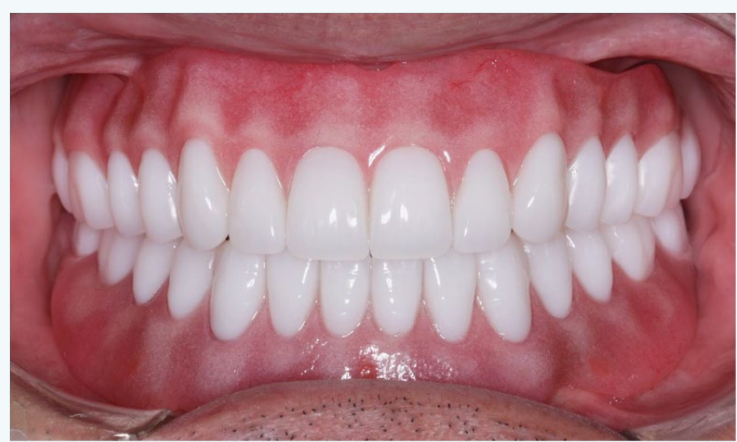

Figure 24B: Maxillary and mandibular over-dentures in-place fully-closed.

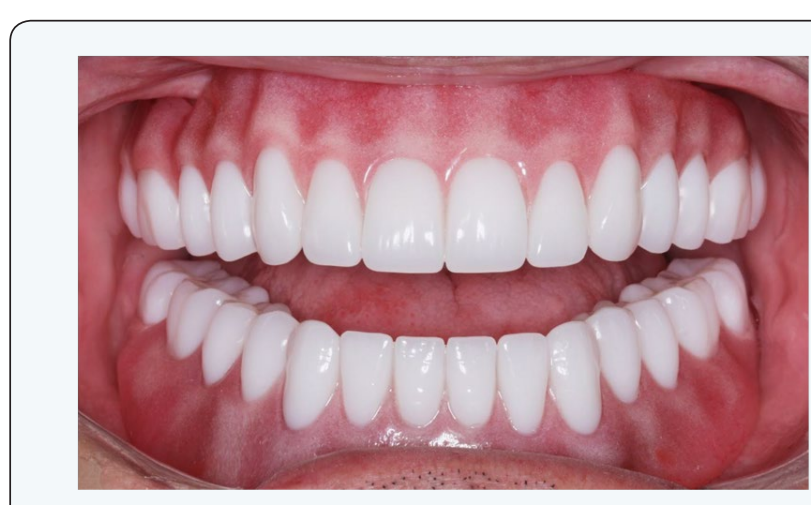

Figure 24C: Maxillary and mandibular over-dentures in place open.

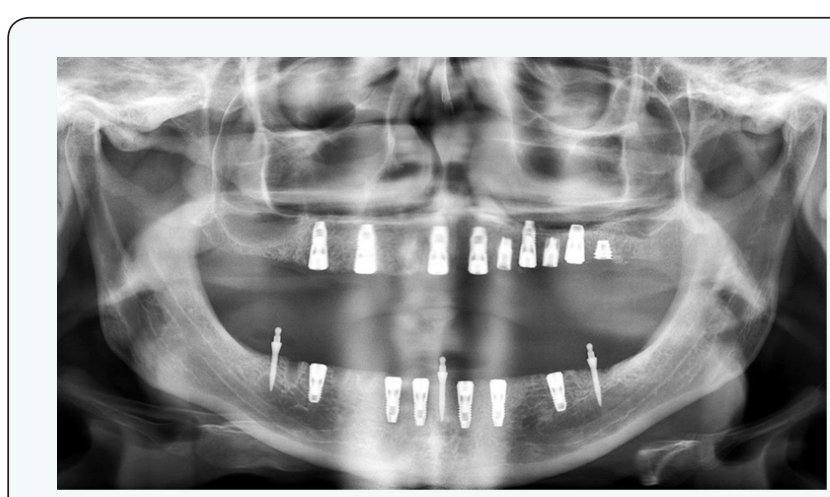

Figure 24D: Panoramic radiograph with reduced implants new implants and mini implants.

\section{Case 3}

Joan is a 73 year old female desiring implant supported over-dentures. She was missing many teeth, and had worn a mandibular removable partial denture for over thirty years. Many of her remaining teeth had failing restorations, long-term poor periodontal prognoses, or had shifted far from their original and ideal positions. Most notably, her maxillary teeth and their previous restorations were unsound and unattractive, and her mandibular teeth had extruded to the point that they were in an un-esthetic "high" position when Joan spoke or smiled, all contributing to her being self-conscious (Figure 25A\&25B). 
A number of restorative options were presented, including conventional dentures, implant over-dentures, and implantsupported hybrid bridges. Keeping and restoring the remaining mandibular teeth was discussed, but the patient did not wish to consider an orthodontic referral to discuss the possibility of tooth intrusion. Full-coverage crowns, while they could make the teeth themselves look better, could not be "intruded" sufficiently for optimal esthetics without prior osseous crown lengthening. In addition, there is a high probability of endodontic issues due to pulpal encroachment when preparing the teeth sufficiently to make the incisal edges of resulting crowns 2-3 mm lower than they currently are [13]. Even if the patient's mandibular teeth were kept and restored, the insufficient alveolar structure in the molar areas to support implants would require a removable partial denture to gain molar function. As a result, the patient opted to have all remaining teeth removed and requested four implants in each arch to support over-dentures. Treatment was initiated by extracting the patient's maxillary teeth and performing an alveoloplasty to smooth and level the remaining osseous ridge prior to implant placement (Figure 26A-26D). Care was given to removing sufficient osseous height to allow room for the necessary layers of materials and components required by an over-denture. These include: 1) teeth, 2) acrylic "gum tissue", 3) strengthening framework and 4) implant LOCATOR abutments. Once the osseous ridge was prepared, the posterior implant positions were sited using previous tooth positions as landmarks. Initial osteotomies were prepared relative to the expected medial wall positions of the sinuses, and paralleling pins were placed with digital radiographs taken to verify the position and angulation. It's worth noting that paralleling pins are significantly narrower than the final implants so care was taken to ensure that there was sufficient room distal to the paralleling pins to allow enlargement of the osteotomies to the appropriate size without perforating the medial sinus walls (Figure 27A-27D). Once the posterior implants were sited, the anterior implants were located in the lateral incisor positions, and their osteotomies were directed toward the palatal aspects of the sockets to engage the thicker, denser portion of the alveolus. All four implants (ASTRA TECH EV by Dentsply, $4.2 \mathrm{x}$ $11 \mathrm{~mm}$ ) were placed at $45 \mathrm{~N} \bullet \mathrm{cm}$, following the manufacturer's protocol of sequentially enlarging the osteotomies, and using copious sterile saline irrigation.

Bone grafting material (Symbios DBX Putty by Dentslpy) was placed in the remaining extraction sites and the flaps were approximated without tension and closed with 4-0 PGA sutures (Hu-Friedy). The maxillary transitional denture was then delivered after relining with Coe-Soft. Four weeks later, the patient's mandibular teeth were extracted and an alveoloplasty was similarly performed to smooth and level the boney ridge in preparation for implant placement. Care was given here as well to removing sufficient osseous height for her future prosthetic needs. For this case, the clinician opted to "lower" her mandibular anterior teeth to return them to their pre-extruded position. The height of the remaining osseous crest was reduced to allow for this (Figure 28A-28F). Following mandibular alveoloplasty, the soft tissue was carefully reflected to visualize the mental foramina, and both posterior implants were sited accordingly. Paralleling pins and digital radiographs were used, as with the maxillary posterior implants, to ensure the enlarging osteotomies would not encroach upon the mental foramina or the anterior loops of the mental nerves. The two anterior implants were then sited appropriately between. All four implants (ASTRA TECH EV by Dentsply, $4.2 \times 11 \mathrm{~mm}$ ) were placed at $45 \mathrm{~N} \bullet \mathrm{cm}$, following the manufacturer's protocol; sequentially enlarging the osteotomies, and with copious sterile saline irrigation. After utilizing the prime mandibular positions for the permanent implants, sites were then selected for the provisional mini implants that would be used to help stabilize the mandibular transitional denture throughout the process. Two $1.8 \times 13 \mathrm{~mm}$ implants (MDI by $3 \mathrm{M}$ ) were placed in the two canine positions and were tightened to a torque value of $40 \mathrm{~N} \bullet \mathrm{cm}$. Bone grafting material (Symbios DBX Putty by Dentsply) was placed in the remaining extraction sites and the flaps were approximated without tension and closed with 4-0 PGA sutures (Hu-Friedy). The O-ring housings were placed on the mini implants and the underside of the transitional denture was hollowed out to allow complete seating on the tissue without binding or impinging on the 0-ring housings. Often, white rubber spacers are used to prevent subsequent acrylic from locking into undercuts should the implants not be perfectly parallel, but were not needed in this case, as the housings were intimate with the soft tissue (Figure 29A-29F). Following application of the associated primer, EZ Pickup (Sterngold) was used to capture the housings into the denture and provide tissue adaptation to the newly sutured gingiva. After curing, excess material was then trimmed and polished away, and the transitional denture was inserted with instructions to leave in place for the first 24 hours to help contain any intra-oral swelling. The patient was instructed to remove the mandibular temporary denture every evening to allow for air to reach the tissue, and also to rinse with Peridex, approximately 2-3 times daily. Three and one-half months were allowed for maxillary implant integration, while two and one-half months were allowed for those in the mandible. All eight implants were uncovered at a single appointment, healing abutments were placed, and the tissue closed with 4-0 PGA sutures. The maxillary transitional denture was readily cleaned out and relined again with Coe-Soft, while the mandibular transitional denture required minor preparation prior to its relining. The hard EZ-Pickup that was used to capture the 0-ring housings previously was largely removed with an acrylic burr, while taking care not to loosen the housings themselves. Coe-Soft was then used to adapt the underside of the transitional denture 
to the new abutments. Peridex use was resumed to help keep the tissue, sutures and abutments clean (Figure 30A-30C). One week later, all sutures were removed and the patient was instructed to start brushing her tissue and abutments to keep them clean, while also stimulating soft tissue blood flow to aid in maturation. Initial impressions were taken and sent to the lab (Root Dental). Another two weeks were allowed for the soft tissue to gain its near-final dimensions prior to final impressions. The healing abutments were removed from all implants and open tray Astra impression pins were placed. Custom trays were used with Aquasil Ultra (Dentsply) for final impressions (Figure 31A-31D). Once final models were fabricated with the appropriate implant abutments, conventional baseplates with wax rims were made and fitted. A subsequent teeth-in-wax try-in was performed to establish ideal vertical dimension and facial form, ideal teeth and smile esthetics, and ideal phonetics. The patient's case was returned to Root Lab where final LOCATOR abutments were selected, strengthening frameworks were cast, and the upper and lower implant over-dentures were processed for final delivery (Figure 32A-32F). At the final delivery appointment, the mini implants were easily removed (unthreaded, without local anesthesia) and the final abutments placed and torqued to Astra's specifications (Figure 33A-33F). Final occlusal balancing was negligible and easily performed to provide the patient proper bilateral group function occlusion. Optimal hygiene was discussed and demonstrated using a Sonicare (Figure 34A-34D).

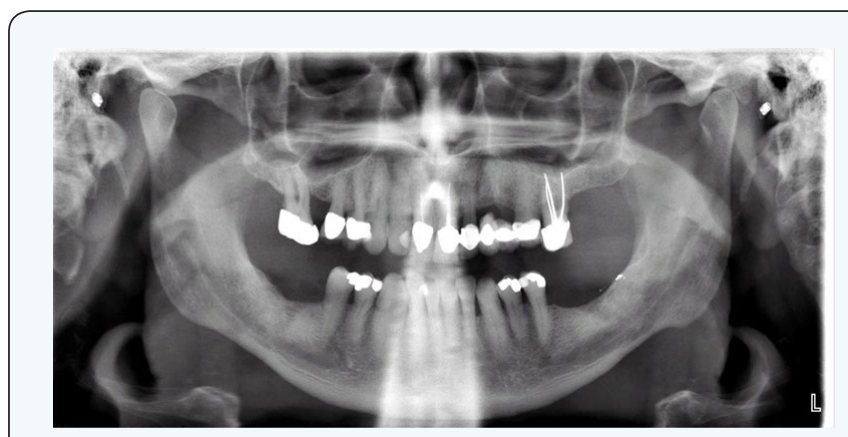

Figure 25A: Preoperative panoramic radiograph.

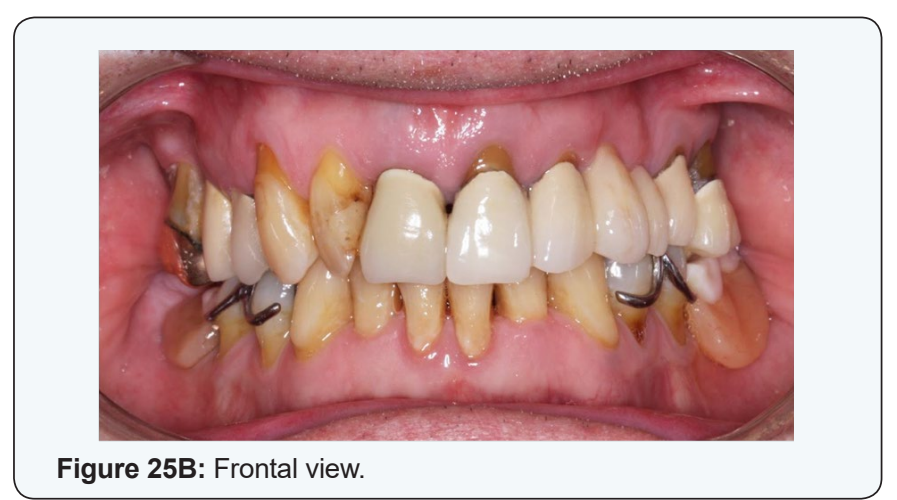

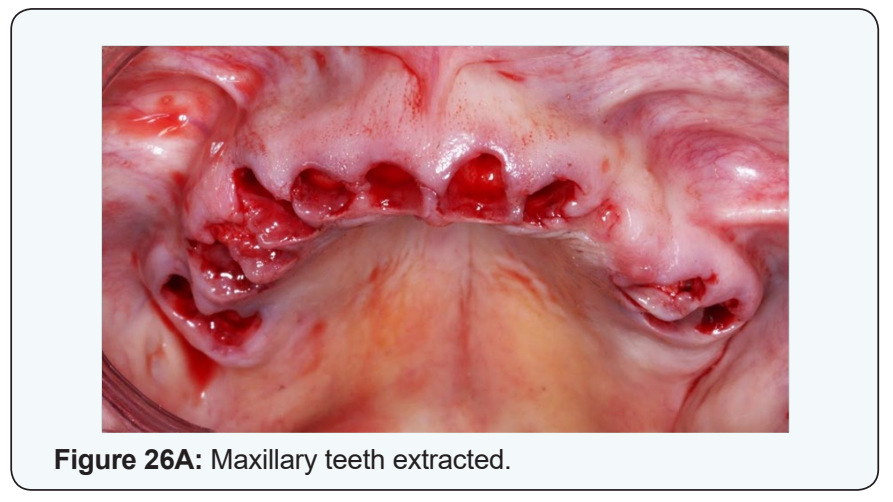
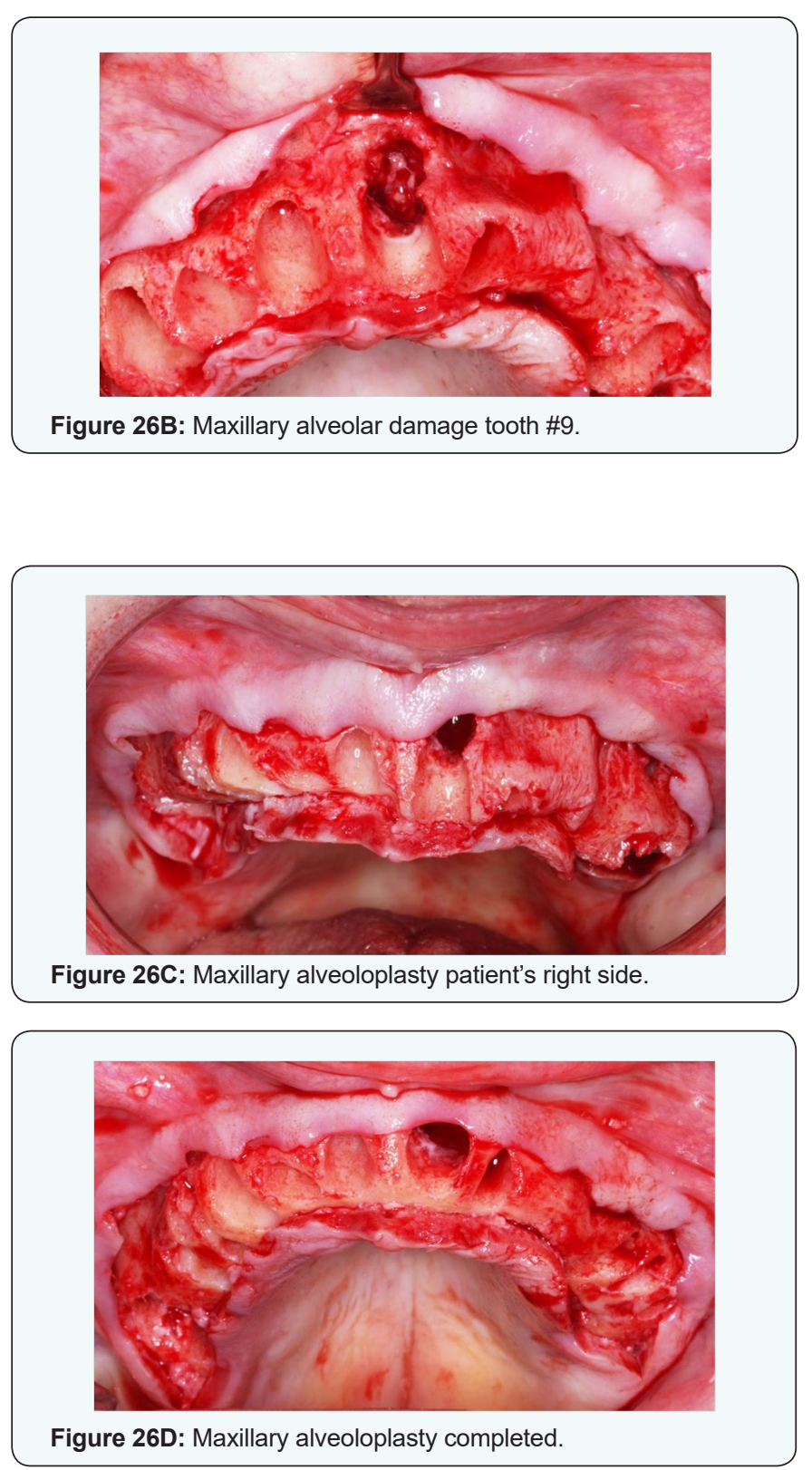


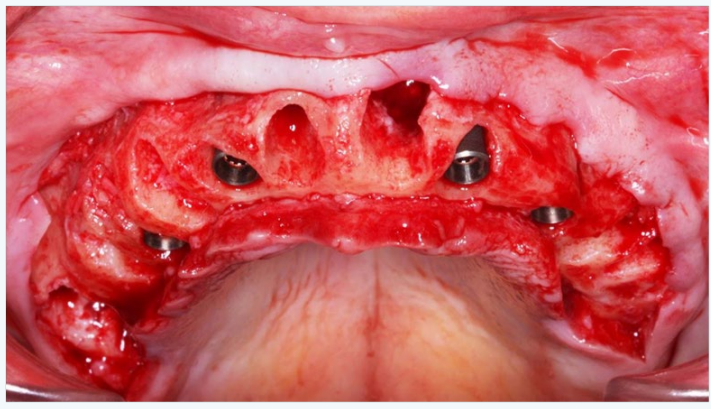

Figure 27A: Maxillary implant placement.

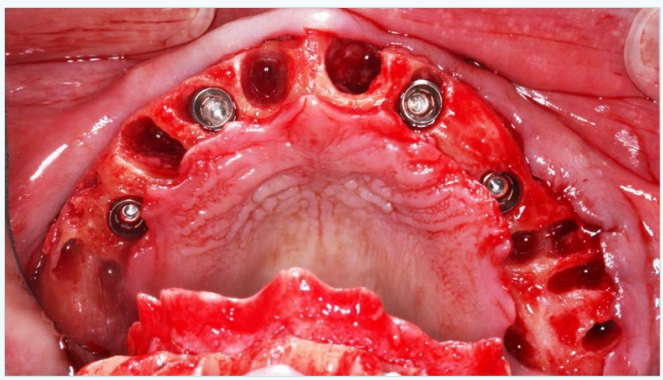

Figure 27B: Maxillary implant placement occlusal view.

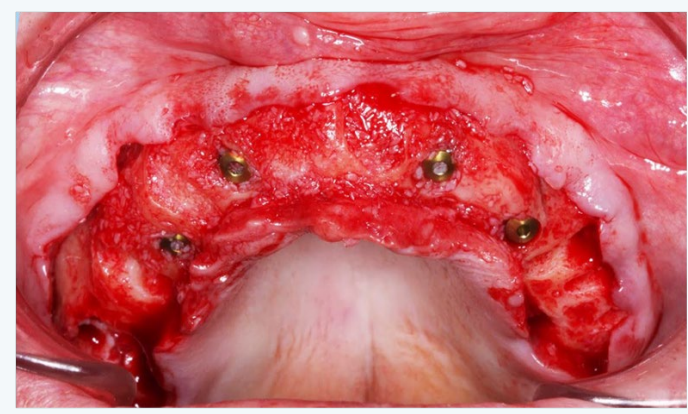

Figure 27C: Maxillary implant placement after socket grafted.

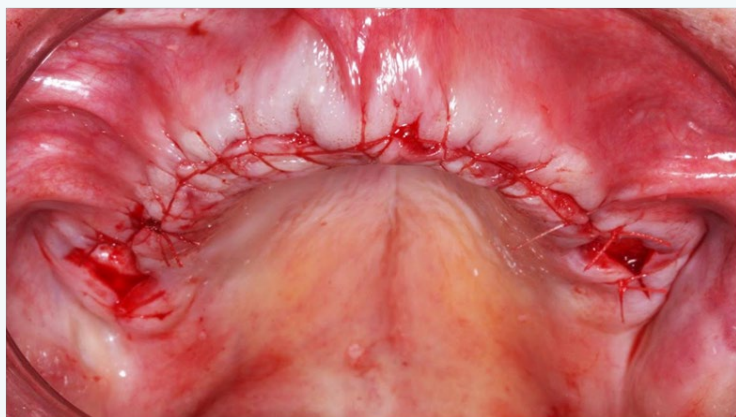

Figure 27D: Maxillary arch sutured.

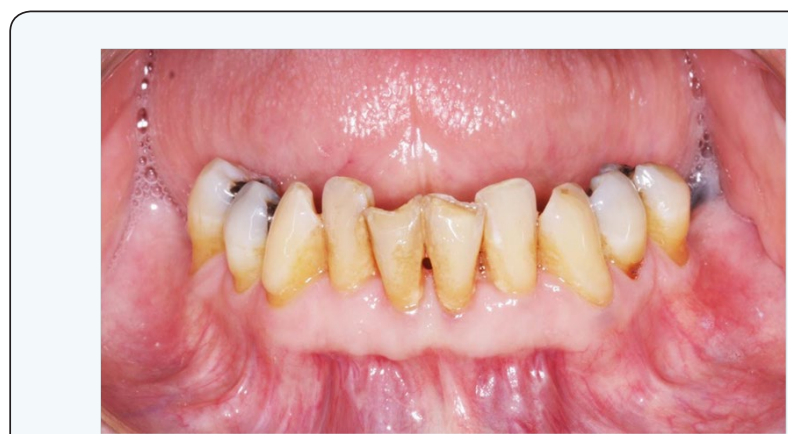

Figure 28A: Mandibular teeth prior to extractions.
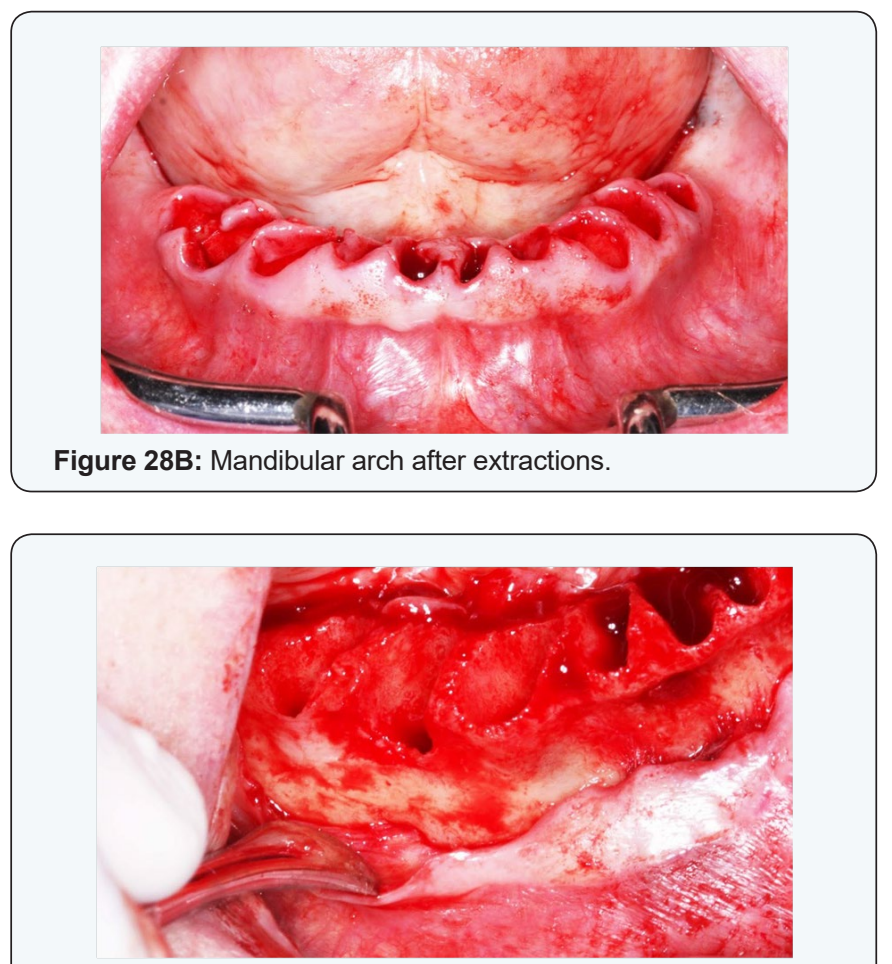

Figure 28C: Mandibular view with right mental nerve appreciated.

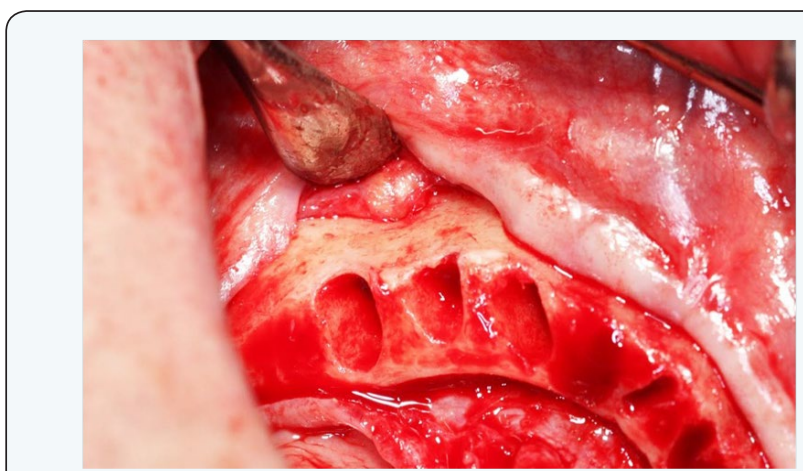

Figure 28D: Mandibular view with left mental nerve appreciated. 


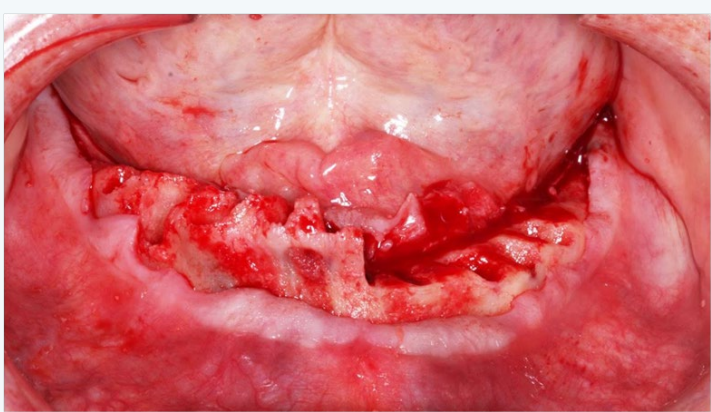

Figure 28E: Mandibular alveoloplasty patient's left side.

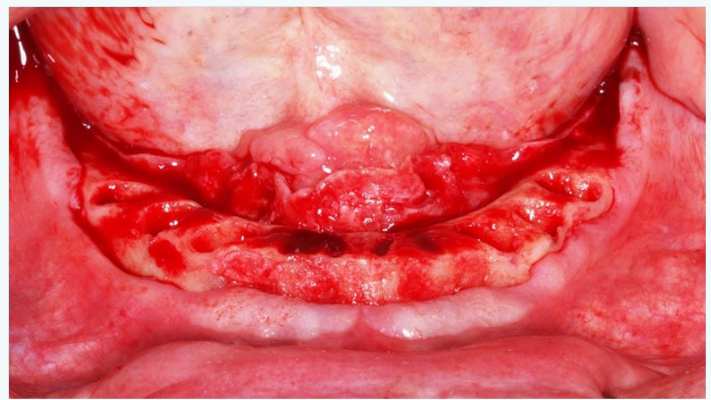

Figure 28F: Mandibular Alveoloplasty Completed.

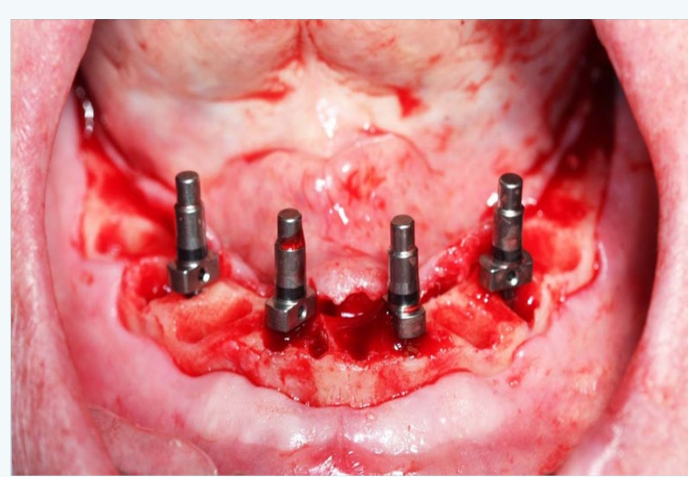

Figure 29A: Mandibular implant positions evaluated for spacing and parallelism.

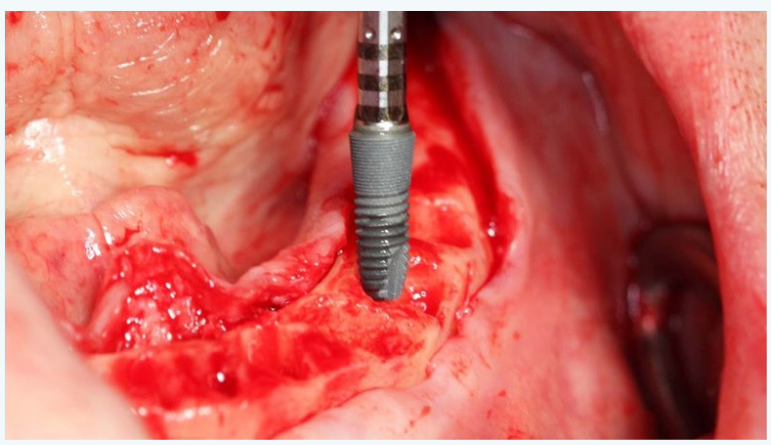

Figure 29B: Insertion of first mandibular implant.

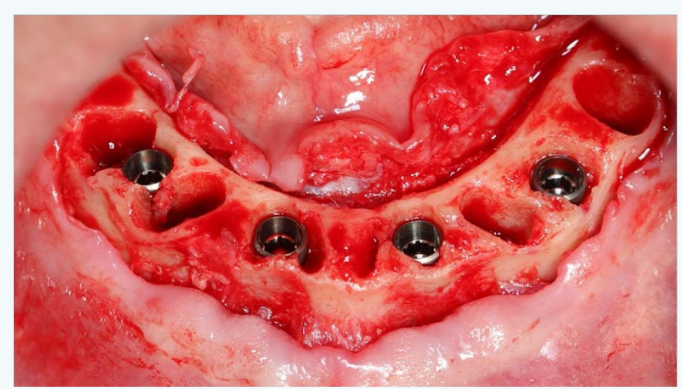

Figure 29C: Mandibular implants placed.

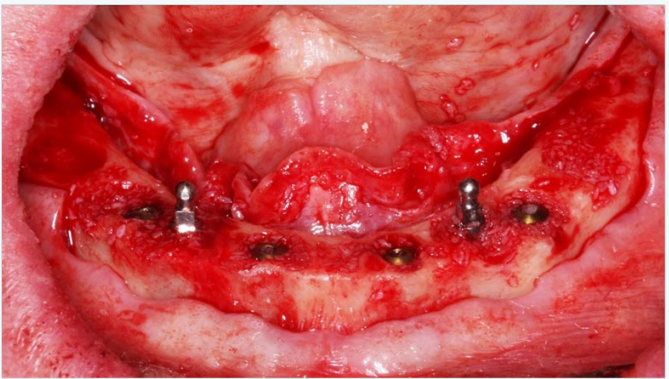

Figure 29D: Mandibular mini-implants placed with bone grafts filling remaining sockets.

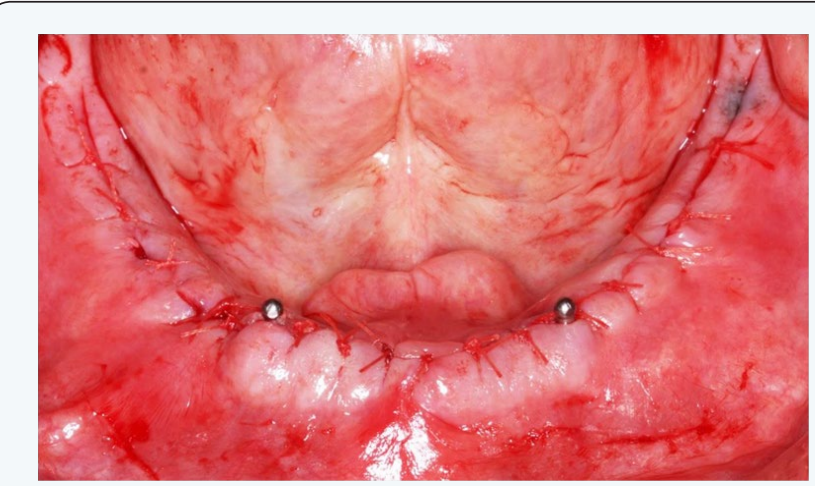

Figure 29E: Mandible sutured with mini-implants exposed.

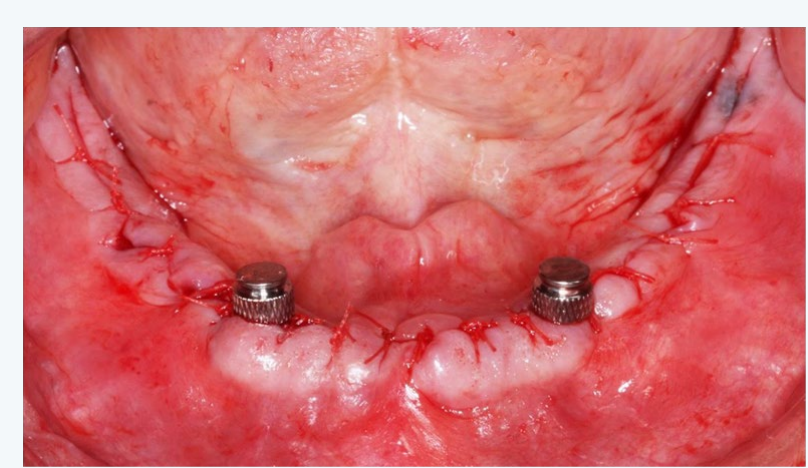

Figure 29F: Retentive housings on mini-implants. 


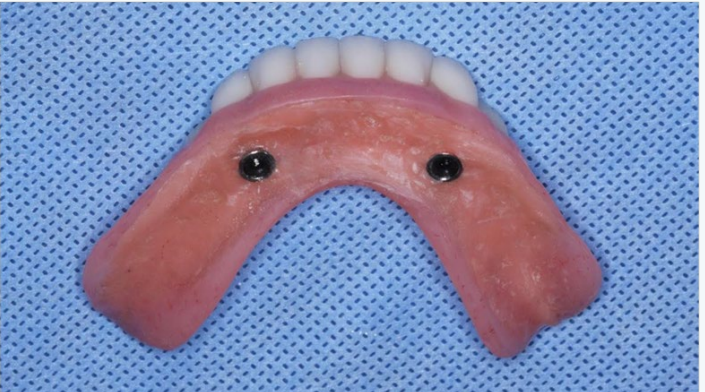

Figure 30A: Intaglio surface of transitional denture after polishing

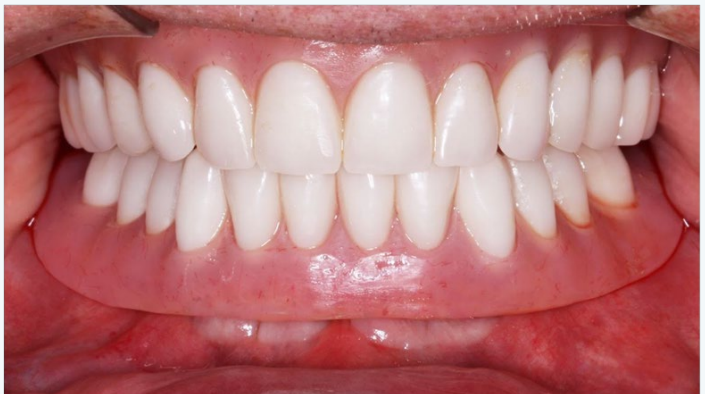

Figure 30B: Maxillary and mandibular transitional dentures placed.

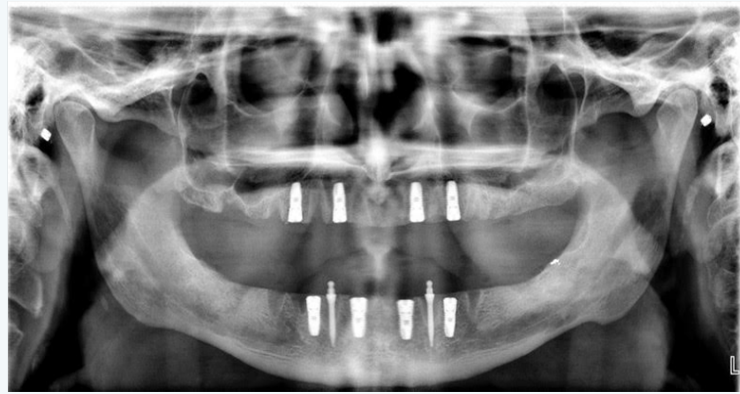

Figure 30C: Postoperative panoramic radiograph.

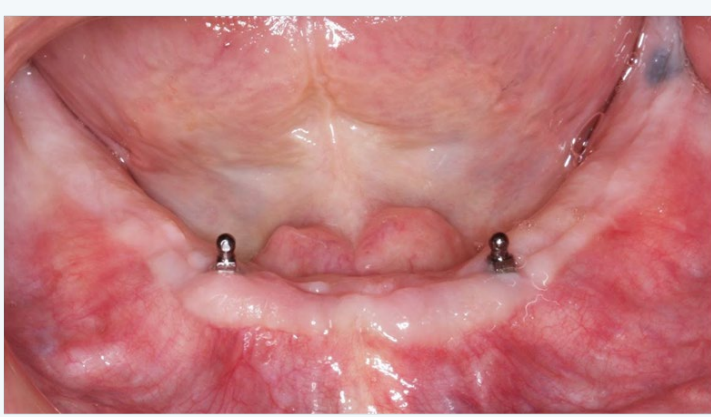

Figure 31A: Mandibular arch with mini-implants.
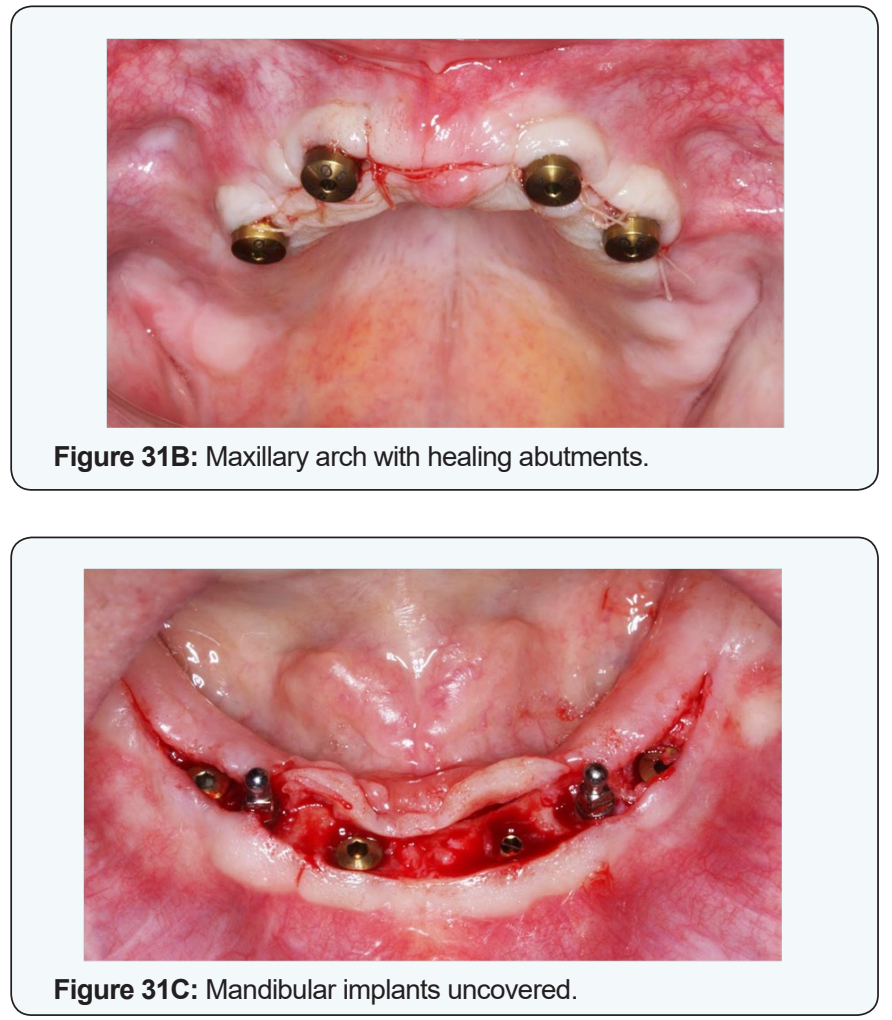

Figure 31C: Mandibular implants uncovered.

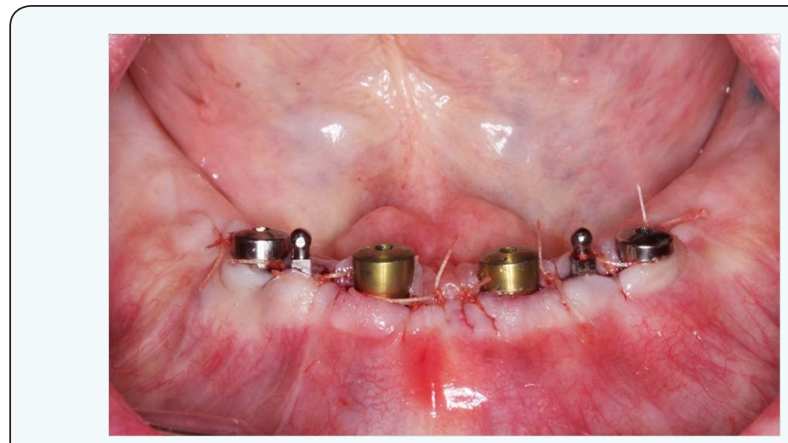

Figure 31D: Mandibular implants with healing abutments placed.

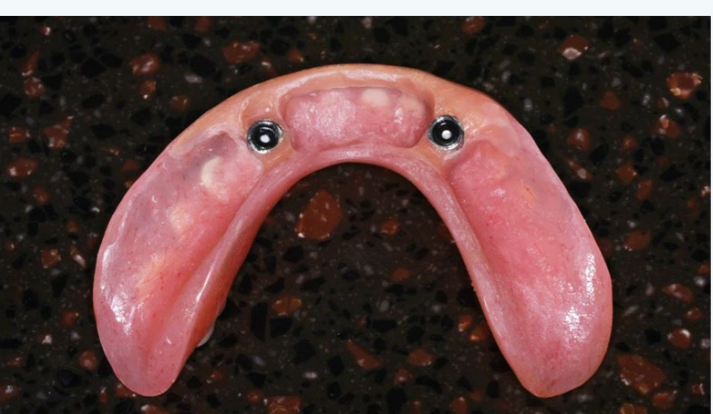

Figure 32A: Mandibular transitional denture intaglio surface prepared. 


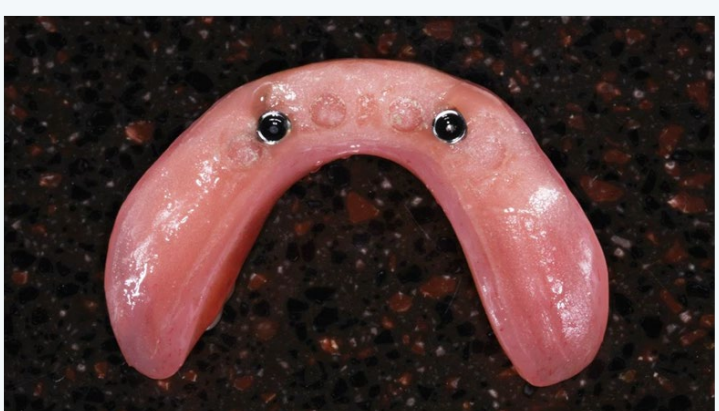

Figure 32B: Mandibular transitional denture with soft liner in place.

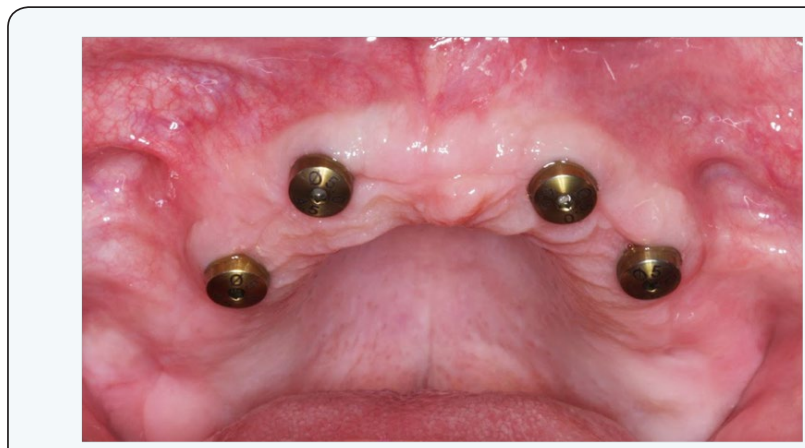

Figure 32C: Maxillary arch with fully-healed implants.

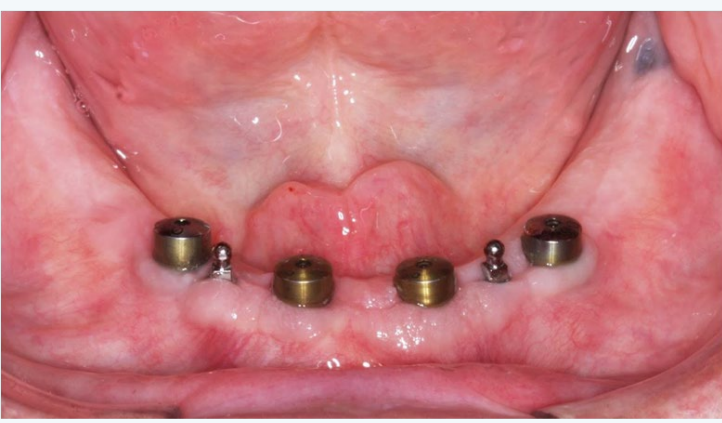

Figure 32D: Mandibular arch with fully-healed implants.

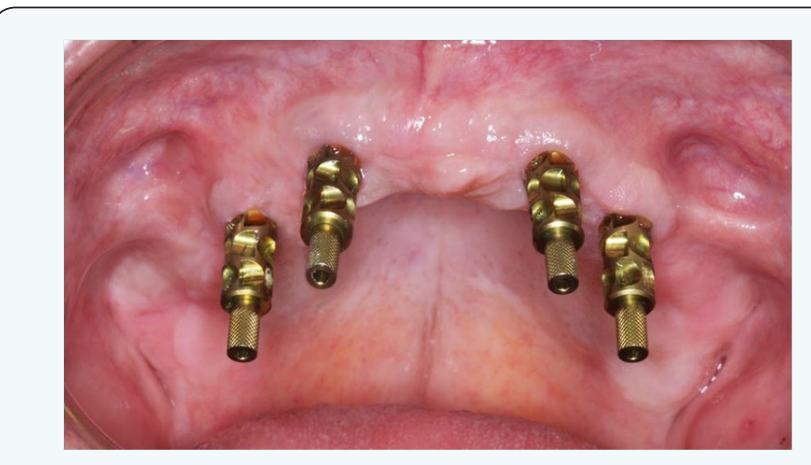

Figure 32E: Maxillary implants with impression abutments placed..

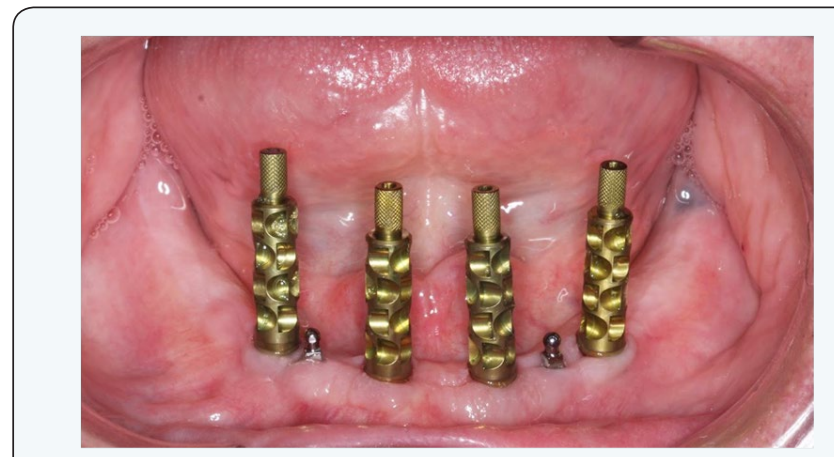

Figure 32F: Mandibular implants with impression abutments placed.
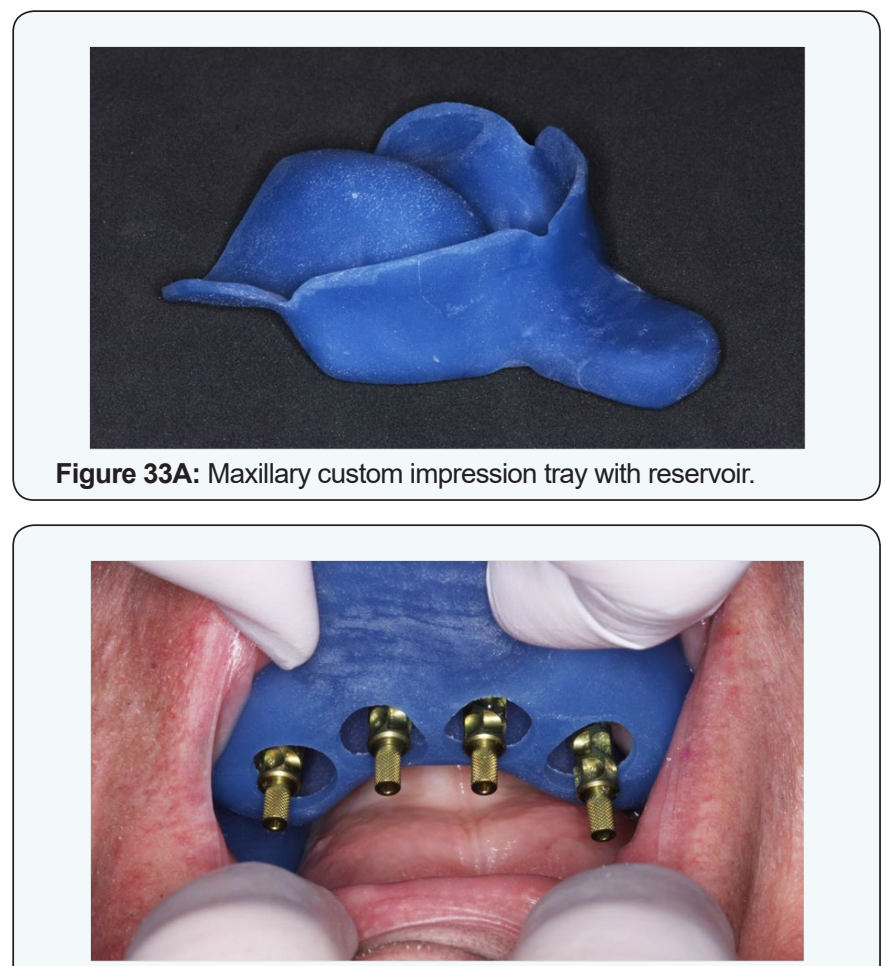

Figure 33B: Maxillary custom impression tray with access openings for impression abutments.

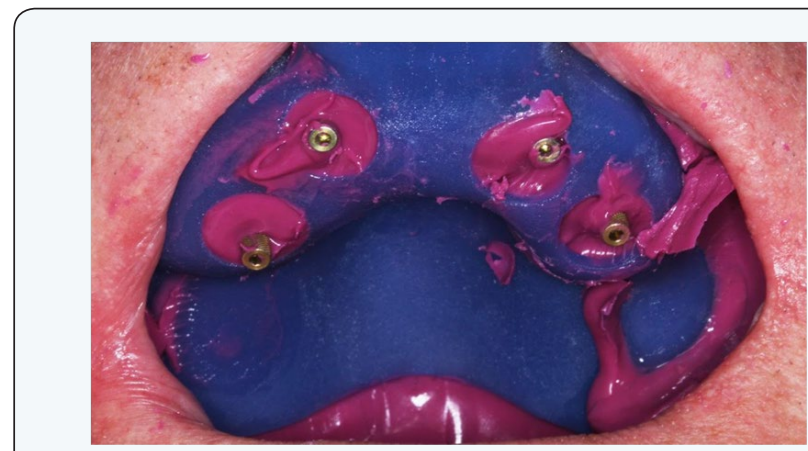

Figure 33C: Maxillary impression tray with impression material capturing impression abutments. 


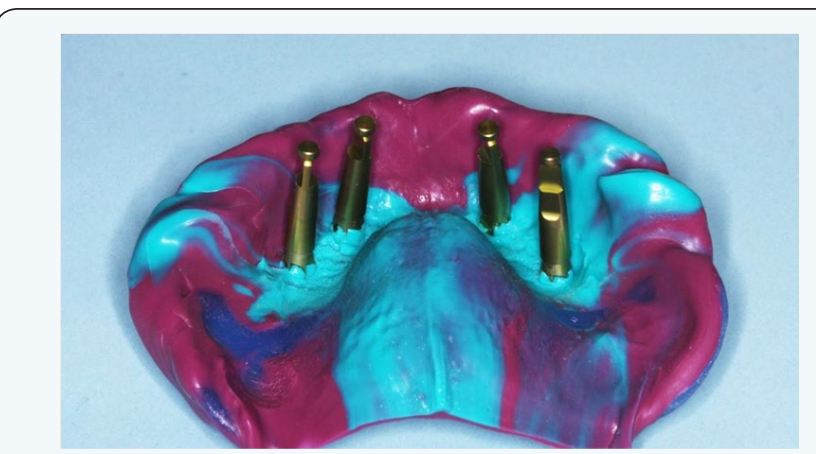

Figure 33D: Maxillary impression with implant analogs placed.
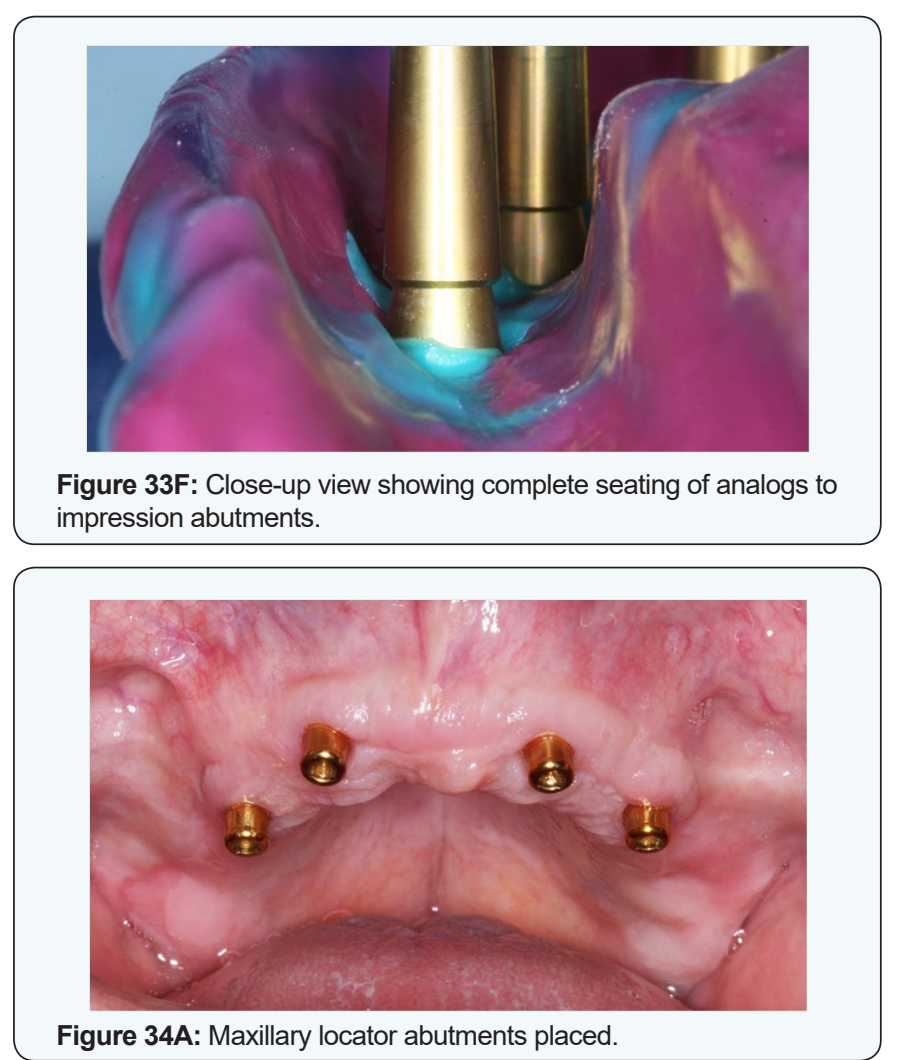

Figure 34A: Maxillary locator abutments placed.

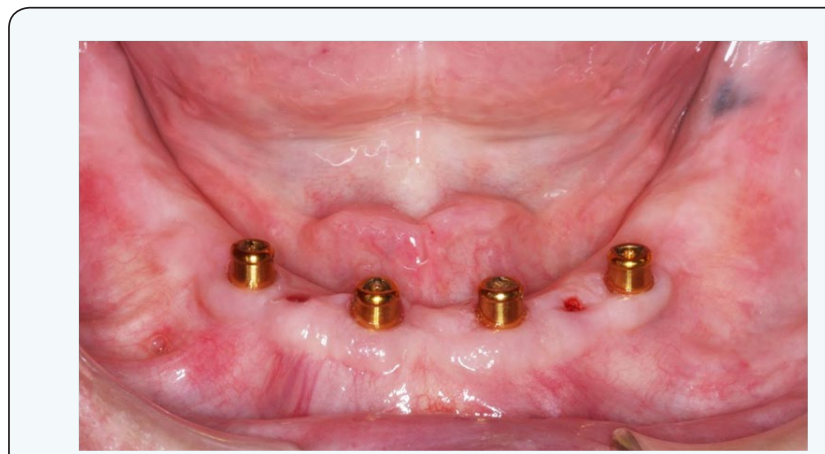

Figure 34B: Mandibular locator abutments placed and miniimplants removed.
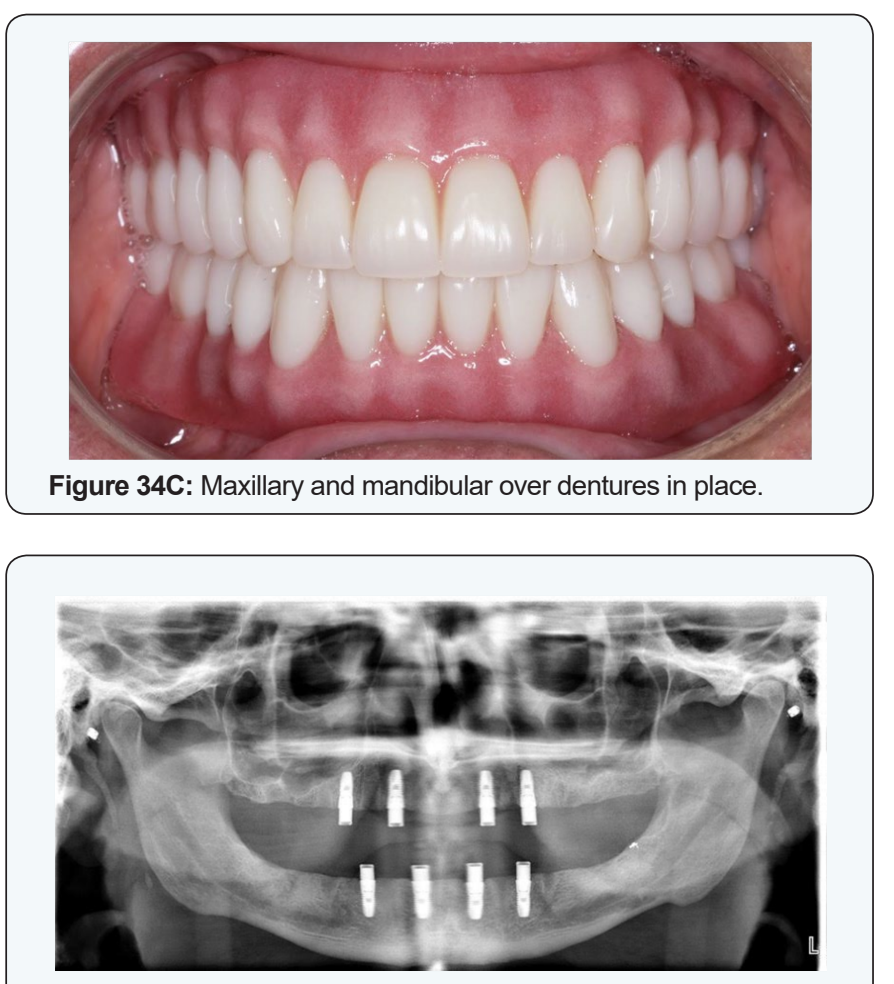

Figure 34D: Final panoramic radiograph.

\section{Summary}

The lack of appropriate and sufficient resources to undertake comprehensive dental treatment plans for the mission patient has been a difficult problem for practitioners over the past thirty years. In addition, the lack of appropriate and consistent treatment time, and the inability to phase treatment over many months, greatly reduces the potential for an optimum outcome. This clinical case report displays a clear-cut, and cost-effective approach that is more serviceable for a wider range of adult patients, than attempting to complete a high-end comprehensive treatment plan. Although clinicians are trained to perform dental treatment that encompasses a wide variety of potential treatments and procedures, the dentist in the mission field has a limited number of treatment options. Proper triage of the patient determines the probable path of treatment, which invariably involves multiple extractions, implant placement and attached complete dentures. Mission clinicians can achieve professionally accepted standards of care for many indigent adult patients without the need for complex restorative treatment.

\section{Acknowledgement}

1. Dentsply, Susquehanna Commerce Center, 221 West Philadelphia Street, York, PA 17401, 1-844-848-0137.

2. Sonicare, 3000 Minuteman Road, M/S 109, Andover, MA 
01810.

3. Sterngold Dental, LLC, 23 Frank Mossberg Drive, Attleboro, MA 02703-0967.

4. Symbios, Susquehanna Commerce Center, 221 West Philadelphia Street, York, PA 17401, Phone: 1-844-8480137.

5. Hu-Friedy Mfg. Co., LLC, 3232 N. Rockwell St., Chicago, IL 60618-5935, 1-800-483-7433.

6. Aquasil, 38 West Clarke Avenue, Milford, DE 19963, 1-800-532-2855.

7. Peridex, 3M Oral Care, 2510 Conway Avenue, St. Paul, MN 55144-1000.

8. Water Pik, Inc, 1730 East Prospect RD, Fort Collins, CO 80553, 1-800-525-2774.

9. Oral-B, 301 E 6th St, Cincinnati, OH 45202, 513-6341111.

10. Kerr Corporation, 1717 West Collins, Orange, CA 92867, 877-685-1484.

11. 3M Oral Care, 2510 Conway Avenue, St. Paul, MN 551441000.

12. Dan Root Dental Laboratories, 5201 College Boulevard, Leawood, KS 66211, 1-800-874-5609.

\section{References}

1. Curro FA, Grill AC, Thompson VP, Craig RG, Vena D, et al. (2011) Advantages of the dental practice-based research network initiative and its role in dental education. J Dent Educ 75(8): 1053-1060.

2. Thompson B, Cooney P, Lawrence H, Ravaghi V, Quinonez C (2014) The potential oral health impact of cost barriers to dental care: findings from a Canadian population-based study BMC Oral Health 14: 78.
3. Muirhead VE, Quinonez C, Figueiredo R, Locker D (2009) Predictors of dental care utilization among working poor Canadians. Community Dent Oral Epidemiol 37(3): 199-208.

4. Horst JA, Clark MD, Lee AH (2009) Observation, assisting, apprenticeship: cycles of visual and kinesthetic learning in dental education. J Dent Educ 73(8): 919-933.

5. Turkyilmaz I, Company AM, McGlumphy EA (2010) Should edentulous patients be constrained to removable complete dentures? The use of dental implants to improve the quality of life for edentulous patients. Gerodontology 27(1): 3-10.

6. Emami E, de Souza RF, Kabawat M, Feine JS (2013) The Impact of edentulism on oral and general health. Int J Dent.

7. Borges Tde F, Mendes FA, de Oliveira TR, Gomes VL, do Prado CJ, et al. (2011) Mandibular overdentures with immediate loading: satisfaction and quality of life. Int J Prosthodont 24(6): 534-539.

8. Schimmel M, Srinivasan M, Herrmann FR, Muller F (2014) Loading protocols for implant-supported overdentures in the edentulous jaw: a systematic review and meta-analysis. Int J Oral Maxillofac Implants 29 Suppl: 271-286.

9. Harris D, Hofer S, O’Boyle CA, Sheridan S, Marley J, et al. (2013) A comparison of implant-retained mandibular overdentures and conventional dentures on quality of life in edentulous patients: a randomized, prospective, within-subject controlled clinical trial. Clin Oral Implants Res 24(1): 96-103.

10. Thomason JM, Lund JP. Chehade A, Feine JS (2003) Patient satisfaction with mandibular implant overdentures and conventional dentures 6 months after delivery. Int J Prosthodont 16(5): 467-473.

11. Kimoto K, Garrett NR (2003) Effect of mandibular ridge height on masticatory performance with mandibular conventional and implantassisted overdentures. Int J Oral Maxillofac Implants 18(4): 523-530.

12. Thomason JM, Heydecke G, Feine JS, Ellis JS (2007) How do patients perceive the benefit of reconstructive dentistry with regard to oral health-related quality of life and patient satisfaction? A systematic review. Clin Oral Implants Res 18 Suppl 3: 168-188.

13. Weber HP, Sukotjo C (2007) Does the type of implant prosthesis affect outcomes in the partially edentulous patient? Int J Oral Maxillofac Implants 22 Suppl: 140-172. 